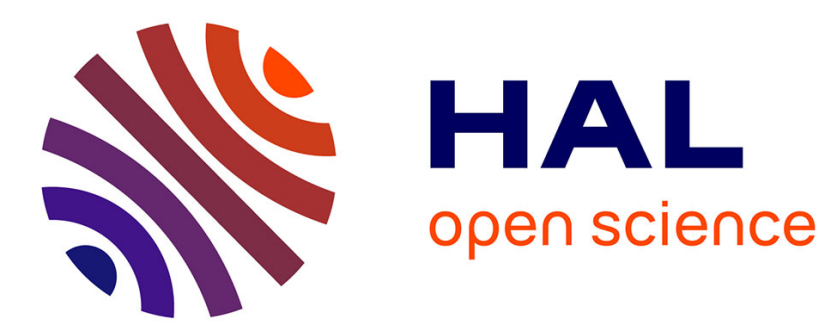

\title{
Convergence of a positive nonlinear DDFV scheme for degenerate parabolic equations
}

El Houssaine Quenjel, Mazen Saad, Mustapha Ghilani, Marianne

Bessemoulin-Chatard

\section{- To cite this version:}

El Houssaine Quenjel, Mazen Saad, Mustapha Ghilani, Marianne Bessemoulin-Chatard. Convergence of a positive nonlinear DDFV scheme for degenerate parabolic equations. Calcolo, In press. hal$01757025 \mathrm{v} 3$

\section{HAL Id: hal-01757025 \\ https://hal.science/hal-01757025v3}

Submitted on 25 May 2020

HAL is a multi-disciplinary open access archive for the deposit and dissemination of scientific research documents, whether they are published or not. The documents may come from teaching and research institutions in France or abroad, or from public or private research centers.
L'archive ouverte pluridisciplinaire HAL, est destinée au dépôt et à la diffusion de documents scientifiques de niveau recherche, publiés ou non, émanant des établissements d'enseignement et de recherche français ou étrangers, des laboratoires publics ou privés. 


\title{
Convergence of a positive nonlinear DDFV scheme for degenerate parabolic equations
}

\author{
El Houssaine Quenjel ${ }^{1,2}$, Mazen Saad ${ }^{2}$, Mustapha Ghilani ${ }^{1}$, and Marianne Bessemoulin-Chatard ${ }^{3}$ \\ ${ }^{1}$ Moulay Ismail University, ENSAM B.P. 15290 EL Mansour 50500 Meknès, Morocco. Email: \\ el-houssaine.quenjel@ec-nantes.fr,m.ghilani@ensam-umi.ac.ma \\ ${ }^{2}$ Ecole Centrale de Nantes, LMJL, CNRS UMR 6629, 1 rue de la Noé, 44321 Nantes, France. Email : \\ mazen.saad@ec-nantes.fr \\ ${ }^{3}$ Université de Nantes, LMJL, CNRS UMR 6629, 2 rue de la Houssinière, 44322 Nantes, France. Email: \\ marianne.bessemoulin@univ-nantes.fr
}

April 27, 2020

\begin{abstract}
In this work, we carry out the convergence analysis of a positive DDFV method for approximating solutions of degenerate parabolic equations. The basic idea rests upon different approximations of the fluxes on the same interface of the control volume. Precisely, the approximated flux is split into two terms corresponding to the primal and dual normal components. Then the first term is discretized using a centered scheme whereas the second one is approximated in a non evident way by an upstream scheme. The novelty of our approach is twofold: on the one hand we prove that the resulting scheme preserves the positivity and on the other hand we establish energy estimates. Some numerical tests are presented and they show that the scheme in question turns out to be robust and efficient. The accuracy is almost of second order on general meshes when the solution is smooth.
\end{abstract}

Keywords. Finite volume scheme, positive, degenerate parabolic equations, convergence.

\section{Problem statement and goal of the paper}

Nonlinear degenerate parabolic equations are the main core to study some complex problems arising, for instance, from petroleum engineering, hydrology and biology. Hence, seeking for analytical or approximate solutions of these equations is of an immense advantage. Throughout this work, we will be interested in approximating, thanks to a new finite volume scheme, the solution to the academic problem:

$$
\left\{\begin{array}{ll}
\partial_{t} u-\nabla \cdot(f(u) \Lambda \nabla u)=0 & \text { in } \quad Q_{T}:=\Omega \times(0, T) \\
u=0 & \text { on } \partial \Omega \times(0, T) \\
u(\cdot, 0)=u^{0} & \text { in } \Omega
\end{array},\right.
$$

where $\Omega$ is a bounded polygonal open of $\mathbb{R}^{d}(d=1,2,3), T$ a fixed positive number, $\partial \Omega$ the boundary of $\Omega, \Lambda$ a given $d$-square matrix (tensor) and $f$ a given nonnegative function. In the context of porous media flows, the function $f$ is usually called the mobility while the tensor $\Lambda$ stands for the permeability. More precisely, the problem (1.1) describes the infiltration of a single fluid through a porous medium with no gravity effects [21]. It is derived from the Darcy law together with the mass conservation equation.

This work is supported by: Ministère de l'Enseignement Supérieur, de la Recherche Scientifique et de la Formation des Cadres du Maroc, CNRST and l'Institut Français au Maroc, and Project MoHyCon - ANR-17-CE40-0027-01 
On the other hand, this problem in known under the name of the porous medium equation [50] whenever $f(u)=u^{m}$, for some nonnegative real number $m$. In view of the theoretical study, the elliptic term of (1.1) can be formulated otherwise by introducing the so-called Kirchhoff transformation $F$. With some general assumptions on $F$, this formulation is sometimes said to be the simplified Stefan problem [30], which is used to model free boundary value problems. Even if this function seems to have no physical interpretation, it will play a remarkable role to carry out the analysis of the scheme we consider here. It is then defined by

$$
F(u)=\int_{0}^{u} f(s) \mathrm{d} s, \quad \forall u \in \mathbb{R} .
$$

In this discretization, we will also introduce the semi-Kirchhoff transform denoted by $\xi$ and defined as

$$
\xi(u)=\int_{0}^{u} \sqrt{f(s)} \mathrm{d} s, \quad \forall u \in \mathbb{R} .
$$

Different approximations, with various assumptions on the data, have been conducted to discretize problems involving nonlinear diffusion equations of type (1.1). For an upstream finite difference method, we cite the work [43]. Concerning finite volume schemes, we refer to this battery of contributions [5, 8, 9, 10, 12, 26, $33,32,35,46]$. Plenty of these discretizations stipulate restrictive constraints, especially an orthogonality condition on the mesh in the sense of Eymard et al. [31], which excludes a large variety of interesting meshes. For example, in Hydrology, most geological layers are quite deformed, then the meshes used to discretize the field should be distorted. In this case, the orthogonality condition can not be satisfied for most of the edges. In addition, in the presence of anisotropic media, we may encounter the same difficulties. Yet, some works have combined finite volume and finite element methods [1, 9, 28, 34, 42, 48]. Carrying out the analysis of these schemes, the authors required a positivity assumption on the stiffness coefficients that does not hold for all sort of meshes. To overcome this issue, positive schemes with their convergence studies have been proposed in [16, 18, 38]. More generally, a gradient scheme [27, 30] has been suggested to discretize the Stefan problem, which is an equivalent formulation of (1.1) using the Kirchhoff transform. The Gradient schemes framework encompasses a lot of popular discretizations, but it may produce undershoots and overshoots in general. There is no hope of proving such bounds without further assumptions.

In this paper, we are concerned with the Discrete Duality Finite Volume (DDFV) method for the discretization of the problem (1.1). This method belongs to the gradient schemes family and is viewed as a particular class of the finite volume methods. It has been first introduced for the Laplace equation in [40, 41]. It has been also proved to be equivalent to a nonconforming finite element approach in [25]. In two dimensions, the convergence analysis of the DDFV scheme was carried out later on for many types of partial differential equations of second order in several works [6, 19, 20, 24, 25]. Such results have been extended to 3D in $[3,4,22,45]$. The strength of this discretization consists of producing a consistent discrete whole gradient on almost general grids and allowing the use of any tensor. This is of a great importance since most of the meshes coming from physics are somehow distorted. On the other hand, the reconstruction gradient operator verifies the discrete Stokes formula, which is a powerful tool to analyze such a scheme. Moreover, the DDFV method is unconditionally coercive, which ensures the stability of the scheme.

Practically (cf. FVCA5 benchmark) [39] the DDFV schemes fail to satisfy an explicit discrete maximum principle. This property is crucial whenever we deal with positive physical quantities by their nature like saturation and concentration. As to be more precise, let us consider the DDFV discretization of the linear diffusion equation $-\Delta u=f$ with Dirichlet boundary conditions. Formally, it yields a stiffness matrix which is not monotone in the case of non admissible meshes in the sense of Eymard et al. [31]. By choosing an appropriate positive source term, we can acquire a solution with some negative values. In general, the monotonicity of the DDFV scheme has been a drawback for the method since it has appeared. However, in the work of [14] the authors were able to design a monotone nonlinear DDFV scheme for the diffusion equation. It basically rests upon the DDFV idea together with a nonlinear monotone two-point finite volume method as investigated in [37, 47, 51]. Unfortunately, there is no convergence proof of the numerical schemes proposed in $[14,37,47,51]$ since they suffer from the lack of coercivity as pointed out in [15, 26]. Recently, in [19] the authors have employed a nonlinear technique to establish the nonnegativity of the 
approximate solution in the case of a linear drift equation enclosed with Neumann boundary conditions. Then, the contribution of our paper is to propose a new scheme that fulfills the physical ranges of the discrete solution even on almost general meshes and for possibly anisotropic tensors. Given an interface of a control volume (primal or dual), the key point of our approach consists of approximating the flux across this interface with a TPFA (Two-Point Flux Approximation) scheme with respect to the unit normal to the same interface and an upwind scheme with respect to the corresponding dual interface. This technique is not standard in the framework of DDFV methods, and it moreover allows to gather the main ingredients to conduct the convergence analysis. From a practical perspective our scheme yields surprising results with optimal convergence rates.

We have chosen to introduce the proposed scheme for degenerate diffusive equations involving homogeneous Dirichlet boundary conditions. The only reason behind the choice of the model problem is the ease readability of our scheme. This approach can be easily extended to more general boundary conditions as done in $[7,25]$ as well as to models including convective and source terms [19, 20,23]. Indeed, the convective term does not provide any supplementary difficulties, since it can be approximated using adequate upstream approaches in order to ensure the discrete maximum principle and get the main elements for the convergence analysis.

The remainder of this paper is structured as follows. In Section 2, we give the DDFV discretization, some related notations and definitions of discrete operators. In Section 3, we sketch out how to derive the proposed DDFV scheme. In Section 4, we prove that this scheme preserves the physical ranges of the approximate solution and we derive some energy estimates on the discrete gradients. In Section 5, we establish that the nonlinear algebraic system has a solution using a monotony criterion. In Section 6, we state some compactness properties and we apply Kolmogorov's theorem to ensure the existence of a convergent subsequence of a family of discrete solutions. In Section 7, we demonstrate that this subsequence tends towards the unique weak solution of the continuous problem. In Section 8, we exhibit some numerical results to show the efficiency and robustness of our scheme.

Let us now formulate the main assumptions on the data.

$\left(\mathrm{A}_{1}\right)$ The initial condition $u^{0}$ is assumed to be in $L^{\infty}(\Omega)$ with $0 \leq u^{0} \leq 1$.

$\left(\mathrm{A}_{2}\right)$ The function $f$ belongs to $\mathcal{C}^{0}([0,1], \mathbb{R})$ with

$$
\left\{\begin{array}{l}
f(u)>0, \quad \text { for all } u \in(0,1), \\
f(u)=0, \quad \text { for all } u \in \mathbb{R} \backslash(0,1) .
\end{array}\right.
$$

As a consequence, $F$ and $\xi$ are Lipschitz continuous nondecreasing functions. We also assume that $v:=\sqrt{f}$ is absolutely continuous. This latter regularity on $v$ is required so that the Engquist-Osher scheme, to be presented later (Section 3), can be defined.

$\left(\mathrm{A}_{3}\right)$ The tensor $\Lambda: \Omega \rightarrow \mathcal{S}_{d}(\mathbb{R})$, where $\mathcal{S}_{d}(\mathbb{R})$ is the space of $d$-square symmetric matrices, is assumed to be in $L^{\infty}(\Omega)^{d \times d}$ and verifies the uniform ellipticity condition

$$
\underline{\Lambda}|\zeta|^{2} \leq \Lambda(x) \zeta \cdot \zeta \leq \bar{\Lambda}|\zeta|^{2} \text {, for all } \zeta \in \mathbb{R}^{d} \text { and a.e. } x \in \Omega,
$$

for some positive constants $\underline{\Lambda}$ and $\bar{\Lambda}$.

We next define the natural space $L^{2}\left(0, T ; H_{0}^{1}(\Omega)\right)$ where the solution to the problem (1.1) will be sought

$$
H_{0}^{1}(\Omega)=\left\{v \in H^{1}(\Omega) / v=0 \text { on } \partial \Omega\right\} .
$$

Moreover, $H_{0}^{1}(\Omega)$ is a Hilbert space endowed with the norm

$$
\|v\|_{H_{0}^{1}(\Omega)}=\|\nabla v\|_{L^{2}(\Omega)^{d}} .
$$

This leads us to the definition of the weak solution. 
Definition 1.1. (Weak solution) A measurable function $u: Q_{T} \longrightarrow[0,1]$ is called a weak solution of the problem (1.1) provided

$$
\begin{aligned}
& \xi(u) \in L^{2}\left(0, T ; H_{0}^{1}(\Omega)\right), \\
& -\int_{Q_{T}} u \partial_{t} \varphi \mathrm{d} x \mathrm{~d} t+\int_{Q_{T}} \Lambda \nabla F(u) \cdot \nabla \varphi \mathrm{d} x \mathrm{~d} t-\int_{\Omega} u^{0} \varphi(\cdot, 0) \mathrm{d} x=0, \quad \forall \varphi \in \mathcal{C}_{c}^{\infty}(\Omega \times[0, T)) .
\end{aligned}
$$

The existence of a weak solution to the problem (1.1) has been investigated in [2] for general parabolic degenerate equatoins, whereas in [21] the authors establish the existence of solutions for two phase immiscible flow in porous media and they also show the maximum principle on the saturation. The uniqueness proof is addressed in [36].

\section{DDFV discretization}

For the simplicity of the exposition, we follow most of the notations given in the works [6, 20]. From now on, we only focus on the two dimensional discretization in space.

\subsection{Meshes and notations}

A DDFV discretization requires three kinds of meshes, a primal mesh, dual mesh and diamond mesh. The primal mesh is denoted by $\overline{\mathfrak{M}}=\mathfrak{M} \cup \partial \mathfrak{M}$, where $\mathfrak{M}$ is a partition of $\Omega$ with polygonal open disjoint subsets usually called control volumes and $\partial \mathfrak{M}$ is the set of boundary edges viewed as degenerate control volumes. These primal grids are not necessarily convex. For every $K \in \overline{\mathfrak{M}}$, the center of gravity of $K$ is denoted by $x_{K}$. We further define $\mathcal{V}$ as the family of these centers.

We designate by $\mathcal{V}^{*}$ the set of all the vertices of the mesh $\mathfrak{M}$. It is composed of inner vertices $\mathcal{V}_{\text {int }}^{*}$ and boundary ones $\mathcal{V}_{e x t}^{*}$. For each $x_{K^{*}} \in \mathcal{V}_{i n t}^{*}$ (resp. $x_{K^{*}} \in \mathcal{V}_{e x t}^{*}$ ), we associate a unique dual control volume $K^{*}$ which is a polygon whose vertices are given by the set $\left\{x_{K} \in \mathcal{V} / x_{K^{*}} \in \bar{K}, K \in \mathfrak{M}\right\}$ (resp. $\left\{x_{K^{*}}\right\} \cup\left\{x_{K} \in \mathcal{V} / x_{K^{*}} \in \bar{K}, K \in \partial \mathfrak{M}\right\}$ ). With these dual sub-domains, we construct the dual mesh denoted by $\overline{\mathfrak{M}^{*}}=\mathfrak{M}^{*} \cup \partial \mathfrak{M}^{*}$ (see Figure 1).

By $\mathcal{E}$ (resp. $\mathcal{E}^{*}$ ) we mean the set of all the edges of $\overline{\mathfrak{M}}$ (resp. $\overline{\mathfrak{M}^{*}}$ ). Two cells are said to be neighbors if they share at least one edge. To be more precise, for every couple of neighboring primal (resp. dual) control volumes $K$ and $L$ (resp. $K^{*}$ and $L^{*}$ ), there exists $\sigma \in \mathcal{E}$ (resp. $\sigma^{*} \in \mathcal{E}^{*}$ ) such that $\sigma=\bar{K} \cap \bar{L}$ (resp. $\left.\sigma^{*}=\overline{K^{*}} \cap \overline{L^{*}}\right)$.

The diamond mesh $\mathfrak{D}=\left(\mathcal{D}_{\sigma, \sigma^{*}}\right)_{\left(\sigma, \sigma^{*}\right) \in \mathcal{E} \times \mathcal{E}^{*}}$ is also a partition of $\Omega$ by diamond cells. For every primal edge $\sigma$ with $\sigma \not \nexists \partial \bar{\Omega}$, the subset $\mathcal{D}_{\sigma, \sigma^{*}}$ is a quadrilateral constructed by connecting the endpoints of $\sigma$ and $\sigma^{*}$. In the case where $\sigma \in \mathcal{E} \cap \partial \bar{\Omega}$, this quadrilateral $\mathcal{D}_{\sigma, \sigma^{*}}$ is nothing more than a triangle as depicted in Figure 2 .

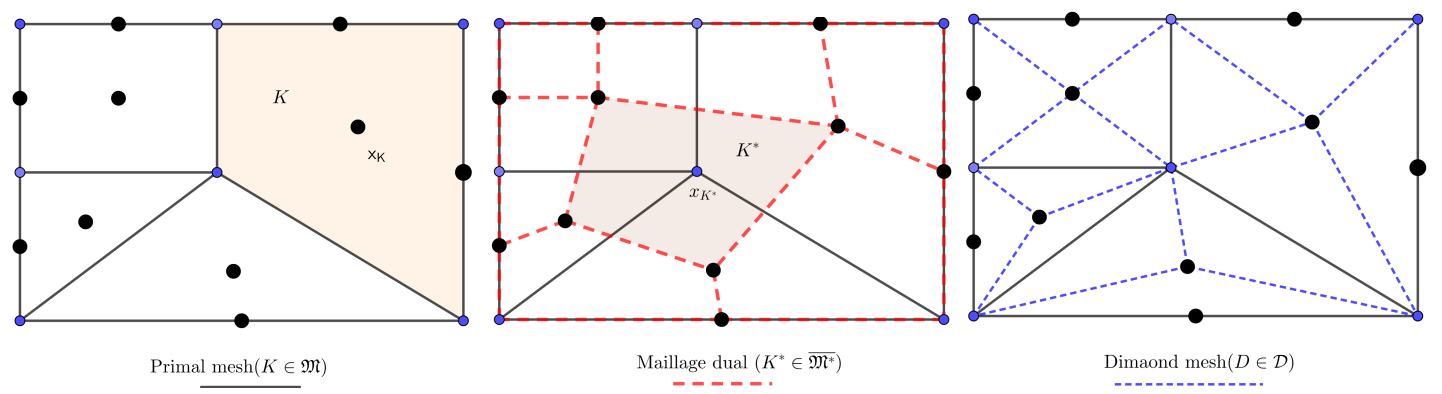

Figure 1: From left to right, the primal mesh, dual mesh and the diamond mesh. 
The DDFV mesh is then given by the union of $\mathcal{T}=\left(\mathfrak{M}, \overline{\mathfrak{M}^{*}}\right)$ and $\mathfrak{D}$. For every $M \in \mathcal{T}$ (primal or dual cell), the notation $m_{M}$ represents the measure of $M, \mathcal{E}_{M}$ contains all the edges of $M, \mathcal{D}_{M}$ is made of all the diamonds $\mathcal{D}_{\sigma, \sigma^{*}}$ such that $m\left(\mathcal{D}_{\sigma, \sigma^{*}} \cap M\right)>0$, and $d_{M}$ refers to the diameter of $M$. For each $\mathcal{D}_{\sigma, \sigma^{*}} \in \mathfrak{D}$, the vertices of $\mathcal{D}_{\sigma, \sigma^{*}}$ are the extremities of both $\sigma$ and $\sigma^{*}$ i.e. $\left(x_{K}, x_{K^{*}}, x_{L}, x_{L^{*}}\right)$. The center $x_{\mathcal{D}}$ of $\mathcal{D}:=\mathcal{D}_{\sigma, \sigma^{*}}$ is defined as the intersection of its main diagonals. $m_{\mathcal{D}}$ stands for the measure of $\mathcal{D}, d_{\mathcal{D}}$ its diameter, and $\alpha_{\mathcal{D}}$ is the angle between $\left(x_{K}, x_{L}\right)$ and $\left(x_{K^{*}}, x_{L^{*}}\right)$. For every edge $e \in \mathcal{E} \cup \mathcal{E}^{*}$, we define $m_{e}$ as its measure. By $\mathbf{n}_{\sigma K}$ (resp. $\mathbf{n}_{\sigma^{*} K^{*}}$ ) we mean the unit normal to $\sigma$ (resp. $\sigma^{*}$ ) outwards $K$ (resp. $K^{*}$ ). Similarly, $\boldsymbol{\tau}_{K, L}$ (resp. $\left.\tau_{K^{*}, L^{*}}\right)$ is the unit tangent vector to $\sigma^{*}(\operatorname{resp} . \sigma)$ oriented from $K$ (resp. $\left.K^{*}\right)$ to $L\left(\operatorname{resp} . L^{*}\right)$.
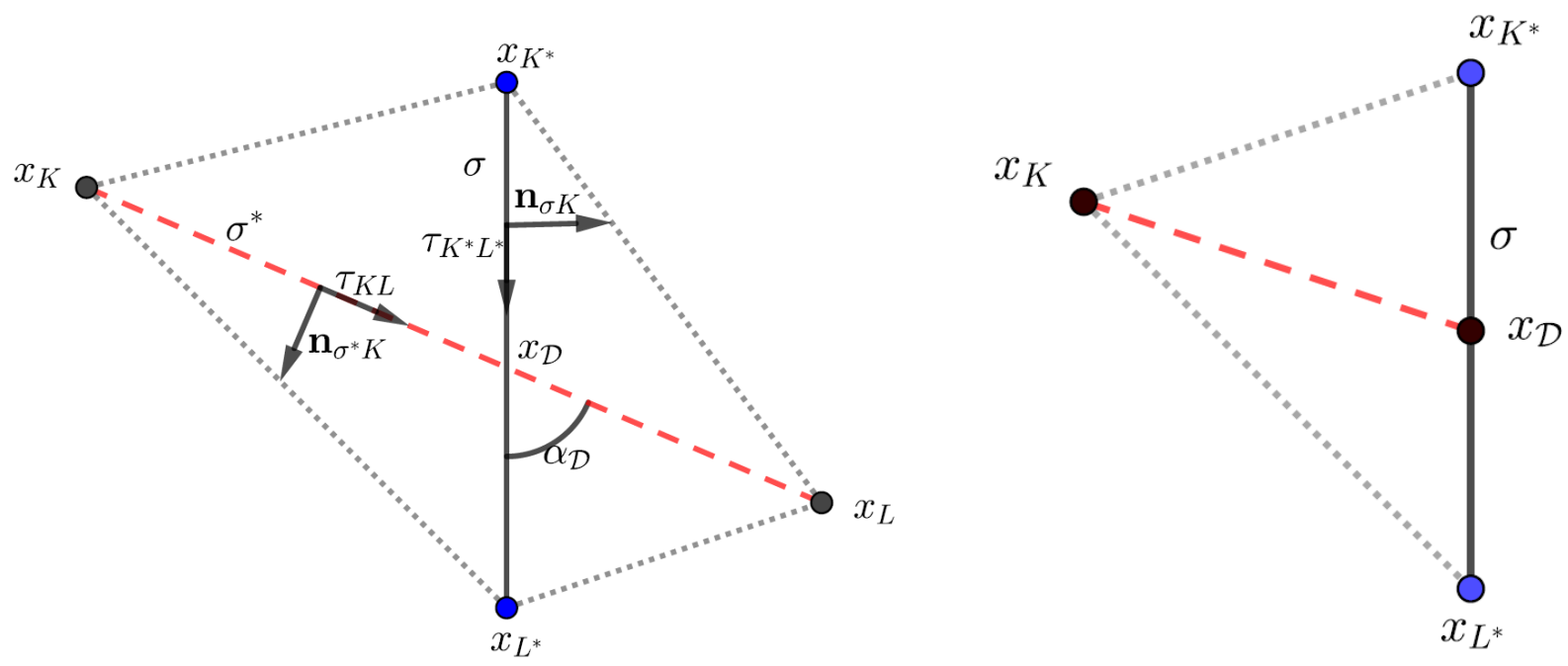

Figure 2: Interior (left) and boundary (right) diamond cells.

Now, we define the regularity of the DDFV mesh that determines how flat the diamond cells are. It also provides information about the difference between the size of a primal (resp. dual) control volume and the size of a diamond cell whenever their intersection is nonempty. This regularity must be controlled as the size of the mesh becomes small in order to perform the convergence analysis of the scheme. Let us denote $h_{\mathfrak{D}}$ the largest diameter of the diamond cells, $\alpha_{\mathcal{T}}$ the unique real number in $\left.] 0, \frac{\pi}{2}\right]$ such that

$$
\sin \left(\alpha_{\mathcal{T}}\right):=\min _{\mathcal{D} \in \mathfrak{D}}\left|\sin \left(\alpha_{\mathcal{D}}\right)\right|
$$

and $\rho_{K}$ (resp. $\left.\rho_{K^{*}}\right)$ the radius of the biggest inscribed ball in $K\left(\right.$ resp. $\left.K^{*}\right)$ whose center is $x_{K}\left(\right.$ resp. $\left.x_{K^{*}}\right)$. Then, the regularity of the mesh is defined by

$$
\begin{array}{r}
\operatorname{reg}(\mathcal{T})=\max \left(\frac{1}{\sin \left(\alpha_{\mathcal{T}}\right)}, \max _{\mathcal{D} \in \mathfrak{D}} \frac{h_{\mathfrak{D}}}{\sqrt{m_{\mathcal{D}}}}, \max _{K \in \mathfrak{M}} \frac{d_{K}}{\sqrt{m_{K}}}, \max _{K^{*} \in \mathfrak{M}^{*}} \frac{d_{K^{*}}}{\sqrt{m_{K^{*}}}},\right. \\
\left.\max _{K \in \overline{\mathfrak{M}}}\left(\frac{d_{K}}{\rho_{K}}+\frac{\rho_{K}}{d_{K}}\right), \max _{K^{*} \in \overline{\mathfrak{M}}^{*}}\left(\frac{d_{K^{*}}}{\rho_{K^{*}}}+\frac{\rho_{K^{*}}}{d_{K^{*}}}\right)\right)
\end{array}
$$

It follows from this relation that there exists a positive constant $C$ depending only on $\operatorname{reg}(\mathcal{T})$ such that

$$
m_{\sigma} m_{\sigma^{*}} \leq C m_{K}, \quad m_{\sigma^{*}}^{2} \leq C m_{\mathcal{D}}, \quad m_{\sigma}^{2} \leq C m_{\mathcal{D}}, \quad m_{\sigma} m_{\sigma^{*}} \leq C m_{\mathcal{D}} .
$$

A time discretization of the interval $(0, T)$ is given by an increasing sequence of real numbers $\left(t^{n}\right)_{n=0, \ldots, N}$ such that

$$
t^{0}=0<t^{1}<\cdots<t^{N}=T .
$$

For every $n \in\{0, \ldots, N-1\}$, we denote $\delta t^{n}=t^{n+1}-t^{n}$ and we define $\delta t=\max _{0 \leq n \leq N-1} \delta t^{n}$. To avoid heavy notations, we assume that the time step $\delta t^{n}$ is uniform. Then $\delta t=\delta t^{n}$, for all $n \in\{0, \ldots, N-1\}$. 


\subsection{Discrete operators}

We now survey the discrete version of the unknowns and operators that will allow us to define the nonlinear DDFV discretization for the problem (1.1). To begin with, let us specify the structure of the space $\mathbb{R}^{\# \mathcal{T}}$. Any vector $u_{\mathcal{T}}$ of this space is written under the form

$$
u_{\mathcal{T}}=\left(\left(u_{K}\right)_{K \in \mathfrak{M}},\left(u_{K^{*}}\right)_{K^{*} \in \overline{\mathfrak{M}^{*}}}\right) .
$$

Next, $\mathbb{R}^{\# \mathcal{T}}$ is endowed by following scalar product

$$
\llbracket u_{\mathcal{T}}, v_{\mathcal{T}} \rrbracket_{\mathcal{T}}=\frac{1}{2}\left(\sum_{K \in \mathfrak{M}} m_{K} u_{K} v_{K}+\sum_{K^{*} \in \overline{\mathfrak{M}^{*}}} m_{K^{*}} u_{K^{*}} v_{K^{*}}\right), \quad \forall u_{\mathcal{T}}, v_{\mathcal{T}} \in \mathbb{R}^{\# \mathcal{T}} .
$$

Additionally, the set $\left(\mathbb{R}^{2}\right)^{\# \mathfrak{D}}$ represents the space of vector fields of the form $\zeta_{\mathfrak{D}}=\left(\zeta_{\mathcal{D}}\right)_{\mathcal{D} \in \mathfrak{D}}$ whose components are basically considered on the diamond cells. This space is endowed by the inner product $(\cdot, \cdot)_{\mathfrak{D}, \Lambda}$ defined as

$$
\left(\zeta_{\mathfrak{D}}, \varphi_{\mathfrak{D}}\right)_{\mathfrak{D}, \Lambda}=\sum_{\mathcal{D} \in \mathfrak{D}} m_{\mathcal{D}} \zeta_{\mathcal{D}} \cdot \Lambda^{\mathcal{D}} \varphi_{\mathcal{D}}, \quad \forall \zeta_{\mathfrak{D}}, \varphi_{\mathfrak{D}} \in\left(\mathbb{R}^{2}\right)^{\# \mathfrak{D}}
$$

where

$$
\Lambda^{\mathcal{D}}=\frac{1}{m_{\mathcal{D}}} \int_{\mathcal{D}} \Lambda(x) \mathrm{d} x, \quad \forall \mathcal{D} \in \mathfrak{D}
$$

\subsubsection{Discrete gradient}

In the framework of the DDFV method, the discrete gradient operator denoted $\nabla^{\mathfrak{D}}$ is a linear mapping from $\mathbb{R}^{\# \mathcal{T}}$ to $\left(\mathbb{R}^{2}\right)^{\# \mathfrak{D}}$. It is defined for every $u_{\mathcal{T}} \in \mathbb{R}^{\# \mathcal{T}}$ by

$$
\nabla^{\mathfrak{D}} u_{\mathcal{T}}=\left(\nabla^{\mathcal{D}} u_{\mathcal{T}}\right)_{\mathcal{D} \in \mathfrak{D}}
$$

where the quantity $\nabla^{\mathcal{D}} u_{\mathcal{T}}$ is referred to as the restriction of the approximate gradient on the diamond cell $\mathcal{D} \in \mathfrak{D}$. On the one hand, for $\mathcal{D} \in \mathfrak{D}$ with $\{\overline{\mathcal{D}} \cap \overline{\partial \Omega}\} \cap \mathcal{E}=\emptyset$, such a restriction is defined so that one can get

$$
\nabla^{\mathcal{D}} u_{\mathcal{T}} \cdot \tau_{K, L}=\frac{u_{L}-u_{K}}{m_{\sigma^{*}}}, \quad \nabla^{\mathcal{D}} u_{\mathcal{T}} \cdot \tau_{K^{*}, L^{*}}=\frac{u_{L^{*}}-u_{K^{*}}}{m_{\sigma}}
$$

or equivalently,

$$
\nabla^{\mathcal{D}} u_{\mathcal{T}}=\frac{1}{\sin \left(\alpha_{\mathcal{D}}\right)}\left(\frac{u_{L}-u_{K}}{m_{\sigma^{*}}} \mathbf{n}_{\sigma K}+\frac{u_{L^{*}}-u_{K^{*}}}{m_{\sigma}} \mathbf{n}_{\sigma^{*} K^{*}}\right) .
$$

On the other hand, our model problem is complemented with Dirichlet boundary conditions. This latter states that the solution is known on $\partial \Omega$. Consequently, for every $\mathcal{D} \in \mathfrak{D}$ with $\overline{\mathcal{D}} \cap \overline{\partial \Omega} \in \mathcal{E}$ (see Figure 2), one has

$$
\nabla^{\mathcal{D}} u_{\mathcal{T}}=\frac{1}{\sin \left(\alpha_{\mathcal{D}}\right)}\left(\frac{u_{\mid \partial \Omega}\left(x_{\mathcal{D}}\right)-u_{K}}{m_{\sigma^{*}}} \mathbf{n}_{\sigma K}+\frac{u_{\mid \partial \Omega}\left(x_{L^{*}}\right)-u_{\mid \partial \Omega}\left(x_{K^{*}}\right)}{m_{\sigma}} \mathbf{n}_{\sigma^{*} K^{*}}\right) .
$$

Notice that the two components of the discrete gradient are reproduced so that one can ensure a consistent approximation of the continuous gradient.

For given $u_{\mathcal{T}}, v_{\mathcal{T}} \in \mathbb{R}^{\# \mathcal{T}}$ and $\mathcal{D} \in \mathfrak{D}$, we define $\delta^{\mathcal{D}} u_{\mathcal{T}}=\left[\begin{array}{c}u_{K}-u_{L} \\ u_{K^{*}}-u_{L^{*}}\end{array}\right]$. Then, one sets

$$
\left(\nabla^{\mathfrak{D}} u_{\mathcal{T}}, \nabla^{\mathfrak{D}} v_{\mathcal{T}}\right)_{\mathfrak{D}, \Lambda}=\sum_{\mathcal{D} \in \mathfrak{D}} \delta^{\mathcal{D}} u_{\mathcal{T}} \cdot \mathbb{A}^{\mathcal{D}, \Lambda} \delta^{\mathcal{D}} v_{\mathcal{T}}
$$

where the local matrix $\mathbb{A}^{\mathcal{D}, \Lambda}$ reads

$$
\mathbb{A}^{\mathcal{D}, \Lambda}=\frac{1}{4 m_{\mathcal{D}}}\left[\begin{array}{cc}
m_{\sigma}^{2} \Lambda^{\mathcal{D}} \mathbf{n}_{\sigma K} \cdot \mathbf{n}_{\sigma K} & m_{\sigma} m_{\sigma^{*}} \Lambda^{\mathcal{D}} \mathbf{n}_{\sigma K} \cdot \mathbf{n}_{\sigma^{*} K^{*}} \\
m_{\sigma} m_{\sigma^{*}} \Lambda^{\mathcal{D}} \mathbf{n}_{\sigma K} \cdot \mathbf{n}_{\sigma^{*} K^{*}} & m_{\sigma^{*}}^{2} \Lambda^{\mathcal{D}} \mathbf{n}_{\sigma^{*} K^{*}} \cdot \mathbf{n}_{\sigma^{*} K^{*}}
\end{array}\right], \quad \forall \mathcal{D} \in \mathfrak{D}
$$


One also defines

$$
\mathbb{A}^{\mathcal{D}}=\frac{1}{4 m_{\mathcal{D}}}\left[\begin{array}{cc}
m_{\sigma}^{2} & m_{\sigma} m_{\sigma^{*}} \mathbf{n}_{\sigma_{K}} \cdot \mathbf{n}_{\sigma^{*} K^{*}} \\
m_{\sigma} m_{\sigma^{*}} \mathbf{n}_{\sigma K} \cdot \mathbf{n}_{\sigma^{*} K^{*}} & m_{\sigma^{*}}^{2}
\end{array}\right], \quad \forall \mathcal{D} \in \mathfrak{D} .
$$

These matrices are positive-definite as given in Lemma A.2. Therefore, the bracket $(\cdot, \cdot)_{\mathfrak{D}, \Lambda}$ is indeed an inner product on $\left(\mathbb{R}^{2}\right)^{\# \mathfrak{D}}$.

In order to make a conspicuous scheme later, we will denote

$$
\begin{aligned}
& a_{K L}:=\frac{1}{\sin \left(\alpha_{\mathcal{D}}\right)} \frac{m_{\sigma}}{m_{\sigma^{*}}} \Lambda^{\mathcal{D}} \mathbf{n}_{\sigma K} \cdot \mathbf{n}_{\sigma K}>0, \quad \eta_{\sigma \sigma^{*}}^{\mathcal{D}}:=\frac{1}{\sin \left(\alpha_{\mathcal{D}}\right)} \Lambda^{\mathcal{D}} \mathbf{n}_{\sigma K} \cdot \mathbf{n}_{\sigma^{*} K^{*}} \in \mathbb{R} \\
& g_{M}:=g\left(u_{M}\right), \quad \forall M \in\left\{K, L, K^{*}, L^{*}\right\} \text { and } g \in\{F, \xi\} \\
& \delta_{L K} u:=u_{L}-u_{K}, \quad \delta_{L^{*} K^{*}} u:=u_{L^{*}}-u_{K^{*}} .
\end{aligned}
$$

\subsubsection{Discrete divergence}

The discrete divergence has been originally introduced in [25] so as to reproduce a discrete counterpart of Green's formula. It is defined by a mapping from $\left(\mathbb{R}^{2}\right)^{\# \mathfrak{D}}$ to $\mathbb{R}^{\# \mathcal{T}}$ as follows:

$$
\operatorname{div}^{\mathcal{T}} \Psi_{\mathfrak{D}}=\left(\operatorname{div}^{\mathfrak{M}} \Psi_{\mathfrak{D}}, \operatorname{div}^{\mathfrak{M}^{*}} \Psi_{\mathfrak{D}}, \operatorname{div}^{\partial \mathfrak{M}^{*}} \Psi_{\mathfrak{D}}\right), \quad \forall \Psi_{\mathfrak{D}}=\left(\Psi_{\mathcal{D}}\right)_{\mathcal{D} \in \mathfrak{D}} \in\left(\mathbb{R}^{2}\right)^{\# \mathfrak{D}}
$$

with $\operatorname{div}^{\mathfrak{M}} \Psi_{\mathfrak{D}}=\left(\operatorname{div}_{K} \Psi_{\mathfrak{D}}\right)_{K \in \mathfrak{M}}, \operatorname{div}^{\mathfrak{M}^{*}} \Psi_{\mathfrak{D}}=\left(\operatorname{div}_{K^{*}} \Psi_{\mathfrak{D}}\right)_{K^{*} \in \mathfrak{M}^{*}}$ and $\operatorname{div}^{\partial \mathfrak{M}^{*}} \Psi_{\mathfrak{D}}=\left(\operatorname{div}_{K^{*}} \Psi_{\mathfrak{D}}\right)_{K^{*} \in \partial \mathfrak{M}^{*}}$ Each component is explicitly given by

$$
\begin{array}{rlrl}
\operatorname{div}_{K} \Psi_{\mathfrak{D}} & =\frac{1}{m_{K}} \sum_{\mathcal{D}_{\sigma, \sigma^{*} \in \mathcal{D}_{K}} m_{\sigma} \Psi_{\mathcal{D}} \cdot \mathbf{n}_{\sigma K},} & \forall K \in \mathfrak{M}, \\
\operatorname{div}_{K^{*}} \Psi_{\mathfrak{D}}=\frac{1}{m_{K^{*}}} \sum_{\mathcal{D}_{\sigma, \sigma^{*} \in \mathcal{D}_{K^{*}}} m_{\sigma^{*}} \Psi_{\mathcal{D}} \cdot \mathbf{n}_{\sigma^{*} K^{*}},} & \forall K^{*} \in \mathfrak{M}^{*}, \\
\operatorname{div}_{K^{*}} \Psi_{\mathfrak{D}}=\frac{1}{m_{K^{*}}}\left(\sum_{\mathcal{D}_{\sigma, \sigma^{*}} \in \mathcal{D}_{K^{*}}} m_{\sigma^{*}} \Psi_{\mathcal{D}} \cdot \mathbf{n}_{\sigma^{*} K^{*}}+\sum_{\mathcal{D}_{\sigma, \sigma^{*}} \in \mathcal{D}_{K^{*} \cap \partial \Omega}} \frac{m_{\sigma^{*}}}{2} \Psi_{\mathcal{D}} \cdot \mathbf{n}_{\sigma^{*} K^{*}}\right), & \forall K^{*} \in \partial \mathfrak{M}^{*}
\end{array}
$$

\subsection{Approximation spaces}

This subsection is devoted to describing the discrete spaces together with some related notations. First, a DDFV mesh is composed of three different partitions. Let us therefore define the discrete functions on these meshes.

(i) We will denote $u_{\mathfrak{M}}$ (resp. $u_{\overline{\mathfrak{M}^{*}}}$ ) the first (resp. second) reconstruction on the primal (resp. dual) mesh, which is a piecewise constant function defined as

$$
u_{\mathfrak{M}}=\sum_{K \in \mathfrak{M}} u_{K} \mathbf{1}_{K}, \quad u_{\overline{\mathfrak{M}^{*}}}=\sum_{K^{*} \in \overline{\mathfrak{M}^{*}}} u_{K^{*}} \mathbf{1}_{K^{*}} .
$$

where $\mathbf{1}_{K}$ is the characteristic function of $K$. We then define the discrete function $u_{h}$ of $L^{1}(\Omega)$ as follows:

$$
u_{h}=\frac{1}{2}\left(u_{\mathfrak{M}}+u_{\overline{\mathfrak{M}^{*}}}\right) .
$$

We henceforth denote $X_{\mathcal{T}}$ the set of all these functions $u_{h}$.

(ii) The third reconstruction $u_{\mathfrak{D}}$ is built on the diamond mesh. It is about piecewise constant functions of the form $u_{\mathfrak{D}}:=\sum_{\mathcal{D} \in \mathfrak{D}} u_{\mathcal{D}} \mathbf{1}_{\mathcal{D}}$ for a given vector $\left(u_{\mathcal{D}}\right)_{\mathcal{D} \in \mathfrak{D}}$. The set of all these functions will be denoted by $X_{\mathfrak{D}}$. 
As a consequence, the time-dependent approximation spaces read:

$$
\begin{aligned}
& X_{\mathcal{T}, \delta t}=\left\{u_{h, \delta t} \in L^{1}\left(Q_{T}\right): u_{h, \delta t}(x, t)=u_{h}^{n+1}(x) / u_{h} \in X_{\mathcal{T}}, \forall t \in\left(t^{n}, t^{n+1}\right], \forall n=0, \cdots, N-1\right\} \\
& X_{\mathfrak{D}, \delta t}=\left\{u_{\mathfrak{D}, \delta t} \in L^{1}\left(Q_{T}\right): u_{\mathfrak{D}, \delta t}(x, t)=u_{\mathfrak{D}}^{n+1}(x) / u_{\mathcal{D}} \in X_{\mathfrak{D}}, \forall t \in\left(t^{n}, t^{n+1}\right], \forall n=0, \cdots, N-1\right\} .
\end{aligned}
$$

For each $u_{h, \delta t} \in X_{\mathcal{T}, \delta t}$, its gradient $\nabla^{\mathcal{D}} u_{h, \delta t} \in X_{\mathfrak{D}, \delta t} \times X_{\mathfrak{D}, \delta t}$ is written by

$$
\nabla^{\mathfrak{D}} u_{h, \delta t}(x, t)=\nabla^{\mathfrak{D}} u_{h}^{n+1}(x), \quad \forall t \in\left(t^{n}, t^{n+1}\right], \forall n=0, \cdots, N-1 .
$$

As for $u_{h, \delta t} \in X_{\mathcal{T}, \delta t}$, we take

$$
u_{\mathfrak{M}, \delta t}(x, t)=u_{\mathfrak{M}}^{n+1}(x), \quad u_{\overline{\mathfrak{M}^{*}}, \delta t}(x, t)=u_{\overline{\mathfrak{M}^{*}}}^{n+1}(x) \quad \forall t \in\left(t^{n}, t^{n+1}\right], \quad \forall n=0, \cdots, N-1 .
$$

Let us now consider a nonlinear function $\mathcal{F}: \mathbb{R} \longrightarrow \mathbb{R}$. We will denote by $\mathcal{F}_{h, \delta t}$ the mean value of $\mathcal{F}\left(u_{\mathfrak{M}, \delta t}\right)$ and $\mathcal{F}\left(u_{\overline{\mathfrak{M}^{*}}, \delta t}\right)$ :

$$
\mathcal{F}_{h, \delta t}=\frac{1}{2}\left(\mathcal{F}\left(u_{\mathfrak{M}, \delta t}\right)+\mathcal{F}\left(u_{\overline{\mathfrak{M}^{*}}, \delta t}\right)\right) .
$$

We next equip the finite dimensional space $X_{\mathcal{T}}$ with the norm $|\cdot|_{p, \mathcal{T}}$. For every $u_{h} \in X_{\mathcal{T}}$, we define

$$
\left|u_{h}\right|_{p, \mathcal{T}}=\left(\frac{1}{2} \sum_{K \in \mathfrak{M}} m_{K}\left|u_{K}\right|^{p}+\frac{1}{2} \sum_{K^{*} \in \overline{M^{*}}} m_{K^{*}}\left|u_{K^{*}}\right|^{p}\right)^{1 / p}, \quad \text { for } \quad 1 \leq p<+\infty
$$

The norm of the discrete gradient is defined by

$$
\left\|\nabla^{\mathfrak{D}} u_{h}\right\|_{p}^{p}=\sum_{\mathcal{D} \in \mathfrak{D}} m_{\mathcal{D}}\left|\nabla^{\mathcal{D}} u_{h}\right|^{p}, \quad \forall 1 \leq p<+\infty .
$$

Observe that

$$
\left\|\nabla^{\mathcal{D}} u_{h}\right\|_{2}^{2}=\sum_{\mathcal{D} \in \mathfrak{D}} \delta^{\mathcal{D}} u_{\mathcal{T}} \cdot \mathbb{A}^{\mathcal{D}} \delta^{\mathcal{D}} u_{\mathcal{T}}
$$

\section{Numerical scheme}

Belonging to the family of finite volume methods, the DDFV scheme is basically obtained by integrating the first equation of (1.1) over $M \times] t^{n}, t^{n+1}$ ], where $M$ is a primal or an internal dual cell. Performing Green's formula yields the balance equation. Then the resulting fluxes are approximated by introducing the definition of the discrete gradient and that of the numerical flux function.

For the convenience of the reader, we briefly look at the discretization of (1.1) on the primal mesh and it is deduced similarly in the case of the dual mesh. So, let $n \in\{0, \ldots, N-1\}$ and $K$ be a primal control volume. Then, one gets

$$
\int_{t^{n}}^{t^{n+1}} \int_{K} \partial_{t} u \mathrm{~d} x \mathrm{~d} t-\sum_{\sigma \in \mathcal{E}_{K}} \int_{t^{n}}^{t^{n+1}} \int_{\sigma} f(u) \Lambda \nabla u \cdot \mathbf{n}_{\sigma K} \mathrm{~d} \sigma \mathrm{d} t=0 .
$$

The evolution term is approximated thanks to the Euler scheme

$$
\int_{t^{n}}^{t^{n+1}} \int_{K} \partial_{t} u \mathrm{~d} x \mathrm{~d} t \approx m_{K}\left(u_{K}^{n+1}-u_{K}^{n}\right),
$$

where $u_{K}^{m}$ is the mean value of $u\left(., t^{m}\right)$ over $K$ for $m=n, n+1$. Concerning the diffusion part, let us remark that from the definitions (1.2) of the Kirchhoff function $F(u)$ and (1.3) of the semi-Kirchhoff function $\xi(u)$ that we have

$$
f(u) \nabla u=\nabla F(u), \quad f(u) \nabla u=v(u) \nabla \xi(u),
$$


with $v(u)=\sqrt{f(u)}$. Then, we propose to approximate the diffusive flux by splitting it into two terms corresponding to the primal and dual normal component of the discrete gradient making use of the two expressions of $f(u) \nabla u$ given above. We thus consider the following discretization

$$
-\int_{t^{n}}^{t^{n+1}} \int_{\sigma} f(u) \Lambda \nabla u \cdot \mathbf{n}_{\sigma K} \mathrm{~d} \sigma \mathrm{d} t \approx \delta t\left(a_{K L}\left(F\left(u_{K}^{n+1}\right)-F\left(u_{L}^{n+1}\right)\right)+v_{K L}^{n+1} \eta_{\sigma \sigma^{*}}^{\mathcal{D}}\left(\xi\left(u_{K^{*}}^{n+1}\right)-\xi\left(u_{L^{*}}^{n+1}\right)\right)\right),
$$

where $v_{K L}^{n+1}$ is an upstream approximation of $v(u)$ on the primal edge $\sigma$. Basically, the first term of (3.3) represents a central discretization of the flux in the direction $\tau_{K, L}$ whereas the second contribution is an upstream approximation of the same flux in the direction $\tau_{K^{*}, L^{*}}$. Since $a_{K L}>0$, the central part is therefore monotone, and the upstream correction is needed to reinforce positivity in the second term since the quantity $\eta_{\sigma \sigma^{*}}^{\mathcal{D}}\left(\xi\left(u_{K_{1}^{*}}^{n+1}\right)-\xi\left(u_{L^{*}}^{n+1}\right)\right)$ is a real number which is not necessarily positive. We next provide the construction of $v_{K L}^{n+1}$. This consists of considering the Engquist-Osher scheme [49], which reads

$$
v_{K L}^{n+1}= \begin{cases}v_{\downarrow}\left(u_{L}^{n+1}\right)+v_{\uparrow}\left(u_{K}^{n+1}\right) & \text { if } \eta_{\sigma \sigma^{*}}^{\mathcal{D}}\left(\xi_{K^{*}}^{n+1}-\xi_{L^{*}}^{n+1}\right) \geq 0 \\ v_{\downarrow}\left(u_{K}^{n+1}\right)+v_{\uparrow}\left(u_{L}^{n+1}\right) & \text { else }\end{cases}
$$

where the functions $v_{\downarrow}, v_{\uparrow}$ are given by

$$
v_{\uparrow}(u):=\int_{0}^{u}\left(v^{\prime}(s)\right)^{+} \mathrm{d} s, \quad v_{\downarrow}(u):=-\int_{0}^{u}\left(v^{\prime}(s)\right)^{-} \mathrm{d} s,
$$

and $x^{+}=\max (x, 0), x^{-}=\max (-x, 0)$ for all $x \in \mathbb{R}$. This convention will be adopted hereafter. In light of hypothesis $\left(A_{2}\right)$, the functions $v_{\uparrow}, v_{\downarrow}$ exist.

The computation of the numerical fluxes will essentially depend on (3.3). For polynomial expressions of $f$, the integrals appearing in $v_{\downarrow}, v_{\uparrow}$, can be obtained straightforwardly; and for more general expressions, we can use an adequate approximation of integrals thanks to prior tables. In the case of general problems exhibiting a complex mobility function $[1,5,12,18]$, an alternative strategy to calculate the fluxes is to consider the following Godunov scheme

$$
v_{K L}^{n+1}=\left\{\begin{array}{ll}
\max _{u \in I_{K L}^{n+1}} v(u) & \text { if } \eta_{\sigma, \sigma *}^{\mathcal{D}}\left(\xi_{K^{*}}^{n+1}-\xi_{L^{*}}^{n+1}\right)\left(u_{K}-u_{L}\right) \geq 0 \\
\min _{u \in I_{K L}^{n+1}} v(u) & \text { otherwise }
\end{array},\right.
$$

where $I_{K L}^{n+1}=\left[\min \left(u_{K}^{n+1}, u_{L}^{n+1}\right), \max \left(u_{K}^{n+1}, u_{L}^{n+1}\right)\right]$. One defines $v_{K^{*} L^{*}}^{n+1}$ in a similar way. Let us however address a remarkable drawback of this choice. First, it is not known how to compute analytically these expressions for general shape functions. This necessitates an external algorithm that determines extrema's of the function $v$ on each interface $K \mid L$ and at every time iteration which could be too expensive and slow the solver.

We wish to emphasize that one can rewrite the quantity $v_{K L}^{n+1} \eta_{\sigma \sigma^{*}}^{\mathcal{D}}\left(\xi_{K^{*}}^{n+1}-\xi_{L^{*}}^{n+1}\right)$ thanks to a numerical flux function $G$ as follows

$$
G\left(u_{K}^{n+1}, u_{L}^{n+1} ; \eta_{\sigma \sigma^{*}}^{\mathcal{D}} \delta_{L^{*} K^{*}}^{n+1} \xi(u)\right)=v_{K L}^{n+1} \eta_{\sigma \sigma^{*}}^{\mathcal{D}}\left(\xi_{K^{*}}^{n+1}-\xi_{L^{*}}^{n+1}\right) .
$$

We recall that a function $G$ of arguments $(a, b, c) \in \mathbb{R}^{3}$ is said to be a numerical flux if the assertions below are satisfied:

$$
\left\{\begin{array}{l}
\left(H_{1}\right) \quad G(\cdot, b, c) \text { is nondecreasing and continuous for all } b, c \in \mathbb{R}, \\
\quad \text { and } G(a, \cdot, c) \text { is nonincreasing and continuous for all } a, c \in \mathbb{R} ; \\
\left(H_{2}\right) \quad G(a, b, c)=-G(b, a,-c) \text { for all } a, b, c \in \mathbb{R} ; \\
\left(H_{3}\right) \quad G(a, a, c)=v(a) c \text { for all } a, c \in \mathbb{R} .
\end{array}\right.
$$

As stressed in $[6,20]$, we require a penalization operator, which is crucial to pass to the limit in the scheme. This penalty term permits to check that the approximate solution on the primal mesh and the dual mesh tend to the same limit. It will be also a key point in our study for the convergence of the diffusive term. 
To this purpose, let $\varepsilon \in] 0,2\left[\right.$ and $u_{\mathcal{T}} \in \mathbb{R}^{\# \mathcal{T}}$. The penalization $\mathcal{P}^{\mathcal{T}}$ is a map from $\mathbb{R}^{\# \mathcal{T}}$ to $\mathbb{R}^{\# \mathcal{T}}$ defined, for all $u_{\mathcal{T}}$, by

$$
\mathcal{P}^{\mathcal{T}} u_{\mathcal{T}}=\left(\mathcal{P}^{\mathfrak{M}} u_{\mathcal{T}}, \mathcal{P}^{\mathfrak{M}^{*}} u_{\mathcal{T}}, \mathcal{P}^{\partial \mathfrak{M}^{*}} u_{\mathcal{T}}\right)
$$

where $\mathcal{P}^{\mathfrak{M}} u_{\mathcal{T}}=\left(\mathcal{P}_{K} u_{\mathcal{T}}\right)_{K \in \mathfrak{M}}, \mathcal{P}^{\mathfrak{M}^{*}} u_{\mathcal{T}}=\left(\mathcal{P}_{K^{*}} u_{\mathcal{T}}\right)_{K^{*} \in \mathfrak{M}^{*}}, \mathcal{P}^{\partial \mathfrak{M}^{*}} u_{\mathcal{T}}=\left(\mathcal{P}_{K^{*}} u_{\mathcal{T}}\right)_{K^{*} \in \partial \mathfrak{M}^{*}}$ such that

$$
\begin{aligned}
\mathcal{P}_{K} u_{\mathcal{T}} & =\frac{1}{m_{K}} \frac{1}{h_{\mathfrak{D}}^{\varepsilon}} \sum_{K^{*} \in \overline{\mathfrak{M}^{*}}} m_{K \cap K^{*}}\left(F\left(u_{K}\right)-F\left(u_{K^{*}}\right)\right), \quad \forall K \in \mathfrak{M}, \\
\mathcal{P}_{K^{*}} u_{\mathcal{T}} & =\frac{1}{m_{K^{*}}} \frac{1}{h_{\mathfrak{D}}^{\varepsilon}} \sum_{K \in \mathfrak{M}} m_{K \cap K^{*}}\left(F\left(u_{K^{*}}\right)-F\left(u_{K}\right)\right), \quad \forall K^{*} \in \overline{\mathfrak{M}^{*}} .
\end{aligned}
$$

Owing to the homogeneous Dirichlet boundary condition, one sets $\mathcal{P}_{K^{*}} u_{\mathcal{T}}=0 \quad \forall K^{*} \in \partial \mathfrak{M}^{*}$. Based on the the following elementary inequality, which a consequence of the Cauchy-Schwarz inequality,

$$
(F(a)-F(b))(a-b) \geq(\xi(a)-\xi(b))^{2}, \quad \forall a, b \in \mathbb{R}
$$

one can check that

$$
\begin{aligned}
\llbracket \mathcal{P} u_{\mathcal{T}}, u_{\mathcal{T}} \rrbracket_{\mathcal{T}} & =\frac{1}{2}\left(\sum_{K \in \mathfrak{M}} m_{K} \mathcal{P}_{K} u_{\mathcal{T}} u_{K}+\sum_{K^{*} \in \overline{\mathfrak{M}^{*}}} m_{K^{*}} \mathcal{P}_{K^{*}} u_{\mathcal{T}} u_{K^{*}}\right) \\
& =\frac{1}{2} \frac{1}{h_{\mathfrak{D}}^{\varepsilon}} \sum_{K^{*} \in \overline{\mathfrak{M}^{*}}} \sum_{K \in \mathfrak{M}} m_{K \cap K^{*}}\left(F\left(u_{K}\right)-F\left(u_{K^{*}}\right)\right)\left(u_{K}-u_{K^{*}}\right) \\
& \geq \frac{1}{2} \frac{1}{h_{\mathfrak{D}}^{\varepsilon}}\left\|\xi\left(u_{\mathfrak{M}}\right)-\xi\left(u_{\overline{\mathfrak{M}^{*}}}\right)\right\|_{L^{2}(\Omega)}^{2}
\end{aligned}
$$

Thanks to the DDFV discretization, an approximate solution for the problem (1.1) is defined as a function $u_{h, \delta t} \in X_{\mathcal{T}, \delta t}$ satisfying the set of equations:

$$
\begin{aligned}
& u_{M}^{0}=\frac{1}{m_{M}} \int_{M} u^{0}(x) \mathrm{d} x, \quad \forall M \in \mathcal{T}, \\
& \frac{m_{K}}{\delta t}\left(u_{K}^{n+1}-u_{K}^{n}\right) \\
& +\sum_{\mathcal{D}_{\sigma, \sigma^{*}} \in \mathcal{D}_{K}}\left(a_{K L}\left(F_{K}^{n+1}-F_{L}^{n+1}\right)+G\left(u_{K}^{n+1}, u_{L}^{n+1} ; \eta_{\sigma \sigma^{*}}^{\mathcal{D}} \delta_{L^{*} K^{*}}^{n+1} \xi(u)\right)\right) \\
& +\gamma m_{K} \mathcal{P}_{K} u_{\mathcal{T}}^{n+1}=0, \quad \forall K \in \mathfrak{M}, \quad n \geq 0, \\
& \frac{m_{K^{*}}}{\delta t}\left(u_{K^{*}}^{n+1}-u_{K^{*}}^{n}\right) \\
& +\sum_{\mathcal{D}_{\sigma, \sigma^{*}} \in \mathcal{D}_{K^{*}}}\left(a_{K^{*} L^{*}}\left(F_{K^{*}}^{n+1}-F_{L^{*}}^{n+1}\right)+G\left(u_{K^{*}}^{n+1}, u_{L^{*}}^{n+1} ; \eta_{\sigma \sigma^{*}}^{\mathcal{D}} \delta_{L K}^{n+1} \xi(u)\right)\right) \\
& +\gamma m_{K^{*}} \mathcal{P}_{K^{*}} u_{\mathcal{T}}^{n+1}=0, \quad \forall K^{*} \in \mathfrak{M}^{*}, \quad n \geq 0 .
\end{aligned}
$$

To take into account the boundary condition, we impose $u_{M}^{n+1}=0$ for all $M \in \partial \mathfrak{M} \cup \partial \mathfrak{M}^{*}$. The coefficient $\gamma$ is a positive parameter. Let us next check that $G$ defines a numerical flux function. This is the object of the following result.

Lemma 3.1. The numerical flux function $G$ satisfies $\left(H_{1}\right),\left(H_{2}\right)$ and $\left(H_{3}\right)$ given in (3.5).

Proof. Observe that items $\left(H_{1}\right),\left(H_{3}\right)$ of (3.5) are direct consequences of the expression of $v_{K L}^{n+1}$ given in (3.4) and the assumption $\left(A_{2}\right)$. It remains to check that the assertion $\left(H_{2}\right)$ holds. To this end, we first point out that the discrete gradient on a fixed diamond, which we recall below, is uniquely defined

$$
\nabla^{\mathcal{D}} u_{\mathcal{T}}=\frac{1}{\sin \left(\alpha_{\mathcal{D}}\right)}\left(\frac{u_{L}-u_{K}}{m_{\sigma^{*}}} \mathbf{n}_{\sigma K}+\frac{u_{L^{*}}-u_{K^{*}}}{m_{\sigma}} \mathbf{n}_{\sigma^{*} K^{*}}\right) .
$$


In other words, we associate to the primal interface $\sigma=K \mid L$ a unique dual interface $\sigma=K^{*} \mid L^{*}$. Now if we permute $K, L$ then $K^{*}, L^{*}$ are automatically permuted, but the coefficient $\eta_{\sigma \sigma^{*}}^{\mathcal{D}}$ keeps the same sign. In particular, this asserts that $\eta_{\sigma \sigma^{*}}^{\mathcal{D}}=\eta_{\sigma^{*} \sigma}^{\mathcal{D}}$. Accordingly

$$
\eta_{\sigma \sigma^{*}}^{\mathcal{D}}\left(\xi_{K^{*}}^{n+1}-\xi_{L^{*}}^{n+1}\right)=-\eta_{\sigma \sigma^{*}}^{\mathcal{D}}\left(\xi_{L^{*}}^{n+1}-\xi_{K^{*}}^{n+1}\right) .
$$

According to this identity and the definition of $v_{K L}^{n+1}$ introduced in (3.4), one finds

$$
v_{K L}^{n+1}=v_{L K}^{n+1} .
$$

Hence

$$
G\left(u_{K}^{n+1}, u_{L}^{n+1}, \eta_{\sigma \sigma^{*}}^{\mathcal{D}}\left(\xi_{K^{*}}^{n+1}-\xi_{L^{*}}^{n+1}\right)\right)=-G\left(u_{L}^{n+1}, u_{K}^{n+1}, \eta_{\sigma \sigma^{*}}^{\mathcal{D}}\left(\xi_{L^{*}}^{n+1}-\xi_{K^{*}}^{n+1}\right)\right) .
$$

Remark 3.1. In the case where $\Lambda=I d$, the coefficient $\eta_{\sigma \sigma^{*}}^{\mathcal{D}}$ measures the flatting of the diamond cells. In particular, if $\eta_{\sigma^{*}}^{\mathcal{D}} \equiv 0$ for all $\mathcal{D}$, meaning that the mesh is orthogonal [31], the above discretization reduces to the pioneer TPFA (Two-Point Flux Approximation) scheme for the problem (1.1) on the primal mesh and on the dual mesh separately. Its convergence analysis can be found in [33].

Remark 3.2. Let us fix the penalty coefficient to $\gamma=0$. According to Lemma 3.1, the above numerical scheme is locally conservative i.e. there exists a unique discrete flux $J_{\mathfrak{D}}^{n+1}$ such that the following relationship holds

$$
\llbracket u_{\mathcal{T}}^{n+1}-u_{\mathcal{T}}^{n}+\delta t \operatorname{div}^{\mathcal{T}} J_{\mathfrak{D}}^{n+1}, \psi_{\mathcal{T}} \rrbracket_{\mathcal{T}}=0, \quad \forall \psi_{\mathcal{T}} \in \mathbb{R}^{\# \mathcal{T}} \text { and } n \geq 0 .
$$

Indeed, the function $J_{\mathfrak{D}}^{n+1}=\left(J_{\mathcal{D}}^{n+1}\right)_{\mathcal{D} \in \mathfrak{D}}$ is defined via its two projections with respect to the primal and dual unit normals. In other words, it is sufficient to set

$$
\begin{aligned}
& J_{\mathcal{D}}^{n+1} \cdot \mathbf{n}_{\sigma K}=\frac{1}{m_{\sigma}}\left(a_{K L}\left(F_{K}^{n+1}-F_{L}^{n+1}\right)+G\left(u_{K}^{n+1}, u_{L}^{n+1} ; \eta_{\sigma \sigma^{*}}^{\mathcal{D}} \delta_{L^{*} K^{*}}^{n+1} \xi(u)\right)\right), \\
& J_{\mathcal{D}}^{n+1} \cdot \mathbf{n}_{\sigma^{*} K^{*}}=\frac{1}{m_{\sigma^{*}}}\left(a_{K^{*} L^{*}}\left(F_{K^{*}}^{n+1}-F_{L^{*}}^{n+1}\right)+G\left(u_{K^{*}}^{n+1}, u_{L^{*}}^{n+1} ; \eta_{\sigma \sigma^{*}}^{\mathcal{D}} \delta_{L K}^{n+1} \xi(u)\right)\right) .
\end{aligned}
$$

As a consequence, $J_{\mathcal{D}}^{n+1}$ is expressed in a unique way thanks to the crucial identity [6]

$$
\sin \left(\alpha_{\mathcal{D}}\right) J_{\mathcal{D}}^{n+1}=\left(J_{\mathcal{D}}^{n+1} \cdot \mathbf{n}_{\sigma K}\right) \boldsymbol{\tau}_{K, L}+\left(J_{\mathcal{D}}^{n+1} \cdot \mathbf{n}_{\sigma^{*} K^{*}}\right) \boldsymbol{\tau}_{K^{*}, L^{*}} .
$$

Finally, (3.13) stems from the definition of the discrete divergence given above and that of the scheme.

\section{$4 \quad L^{\infty}$-bound and a priori estimates}

In this section, we show that any solution to the equations of the proposed scheme verifies an $L^{\infty}$-bound. In addition, some a priori estimates are derived on the discrete gradient of the Kirchhoff function. These materials are of importance to prove the convergence of the scheme.

\subsection{Boundedness of discrete solutions}

Lemma 4.1. We assume that $0 \leq u^{0} \leq 1$. For each fixed integer $0 \leq n \leq N-1$, let $\left(u_{\mathcal{T}}^{n+1}\right)$ be a vector of $\mathbb{R}^{\# \mathcal{T}}$ such that the DDFV scheme (3.10)-(3.12) holds. Then, $u_{\mathfrak{M}^{n}}^{n+1}, u_{\mathfrak{M}^{*}}^{n+1}$ belong to $[0,1]$.

Proof. The proof is carried out by induction on $n$. Fix $n \in\{0, \cdots, N-1\}$. Let us assume that the claim is true for $u_{\mathfrak{M}}^{n}, u_{\mathfrak{M}^{*}}^{n}$ and check that it is so for $u_{\mathfrak{M}^{n}}^{n+1}, u_{\mathfrak{M}^{*}}^{n+1}$. To this purpose, we perform the proof in two steps .

Step 1: We consider $u_{K}^{n+1}=\min _{L \in \mathfrak{M}}\left(u_{L}^{n+1}\right)$. Multiplying (3.11) by $-\left(u_{K}^{n+1}\right)^{-}$yields

$$
\begin{gathered}
-\frac{m_{K}}{\delta t}\left(u_{K}^{n+1}-u_{K}^{n}\right)\left(u_{K}^{n+1}\right)^{-}-\sum_{\mathcal{D}_{\sigma, \sigma^{*} \in \mathcal{D}_{K}}}\left(a_{K L}\left(F_{K}^{n+1}-F_{L}^{n+1}\right)+G\left(u_{K}^{n+1}, u_{L}^{n+1} ; \eta_{\sigma \sigma^{*}}^{\mathcal{D}} \delta_{L^{*} K^{*}}^{n+1} \xi(u)\right)\right)\left(u_{K}^{n+1}\right)^{-} \\
-\gamma m_{K} \mathcal{P}_{K} u_{\mathcal{T}}^{n+1}\left(u_{K}^{n+1}\right)^{-}=0 .
\end{gathered}
$$


Since $F$ is a nondecreasing function, we obtain $a_{K L}\left(F_{K}^{n+1}-F_{L}^{n+1}\right) \leq 0$. Furthermore

$$
G\left(u_{K}^{n+1}, u_{L}^{n+1} ; \eta_{\sigma \sigma^{*}}^{\mathcal{D}} \delta_{L^{*} K^{*}}^{n+1} \xi(u)\right)\left(u_{K}^{n+1}\right)^{-} \leq 0 .
$$

Indeed, if $0 \leq u_{K}^{n+1}$ then $\left(u_{K}^{n+1}\right)^{-}=0$. Otherwise, we use the fact that the numerical flux function is nonincreasing with respect to the second argument and that it is consistent

$$
\begin{aligned}
G\left(u_{K}^{n+1}, u_{L}^{n+1} ; \eta_{\sigma \sigma^{*}}^{\mathcal{D}} \delta_{L^{*} K^{*}}^{n+1} \xi(u)\right)\left(u_{K}^{n+1}\right)^{-} & \leq G\left(u_{K}^{n+1}, u_{K}^{n+1} ; \eta_{\sigma \sigma^{*}}^{\mathcal{D}} \delta_{L^{*} K^{*}}^{n+1} \xi(u)\right)\left(u_{K}^{n+1}\right)^{-} \\
& =-v\left(u_{K}^{n+1}\right) \eta_{\sigma \sigma^{*}}^{\mathcal{D}} \delta_{L^{*} K^{*}}^{n+1} \xi(u)\left(u_{K}^{n+1}\right)^{-}=0 .
\end{aligned}
$$

The previous equality holds thanks to the degeneracy of the function $v$ on $]-\infty, 0]$. Let us next demonstrate that

$$
-m_{K} \mathcal{P}_{K} u_{\mathcal{T}}^{n+1}\left(u_{K}^{n+1}\right)^{-} \geq 0
$$

It follows from the definition of the penalization term highlighted in (3.6) that

$$
\begin{aligned}
-m_{K} \mathcal{P}_{K} u_{\mathcal{T}}^{n+1}\left(u_{K}^{n+1}\right)^{-} & =\frac{1}{h_{\mathfrak{D}}^{\varepsilon}} \sum_{K^{*} \in \overline{\mathfrak{M}^{*}}} m_{K \cap K^{*}}\left(-F\left(u_{K}^{n+1}\right)\left(u_{K}^{n+1}\right)^{-}+F\left(u_{K^{*}}^{n+1}\right)\left(u_{K}^{n+1}\right)^{-}\right) \\
& =\frac{1}{h_{\mathfrak{D}}^{\varepsilon}} \sum_{K^{*} \in \overline{\mathfrak{M}}^{*}} m_{K \cap K^{*}} F\left(u_{K^{*}}^{n+1}\right)\left(u_{K}^{n+1}\right)^{-} .
\end{aligned}
$$

We used the fact that $F$ is extended by 0 on $(-\infty, 0)$ to see that $F\left(u_{K}^{n+1}\right)\left(u_{K}^{n+1}\right)^{-}=0$. Since $F\left(u_{K^{*}}^{n+1}\right) \geq 0$, regardless the sign of $u_{K^{*}}^{n+1}$, inequality (4.1) holds. Whence

$$
-\left(u_{K}^{n+1}-u_{K}^{n}\right)\left(u_{K}^{n+1}\right)^{-}=\left|\left(u_{K}^{n+1}\right)^{-}\right|^{2}+\left(u_{K}^{n+1}\right)^{-} u_{K}^{n} \leq 0,
$$

which implies, using the induction assumption, that $\left(u_{K}^{n+1}\right)^{-}=0$. Hence, $u_{K}^{n+1} \geq 0$.

Step 2 : We here switch the role of the control volume $K$ and take now $u_{K}^{n+1}=\max _{L \in \mathfrak{M}}\left(u_{L}^{n+1}\right)$. Multiplying (3.11) by $\left(u_{K}^{n+1}-1\right)^{+}$gives

$$
\begin{aligned}
\frac{m_{K}}{\delta t}\left(u_{K}^{n+1}-u_{K}^{n}\right)( & \left.u_{K}^{n+1}-1\right)^{+} \\
& +\sum_{\mathcal{D}_{\sigma, \sigma^{*} \in \mathcal{D}_{K}}}\left(a_{K L}\left(F_{K}^{n+1}-F_{L}^{n+1}\right)+G\left(u_{K}^{n+1}, u_{L}^{n+1} ; \eta_{\sigma \sigma^{*}}^{\mathcal{D}} \delta_{L^{*} K^{*}}^{n+1} \xi(u)\right)\right)\left(u_{K}^{n+1}-1\right)^{+} \\
& \quad+\gamma m_{K} \mathcal{P}_{K} u_{\mathcal{T}}^{n+1}\left(u_{K}^{n+1}-1\right)^{+}=0
\end{aligned}
$$

It is now evident that $a_{K L}\left(F_{K}^{n+1}-F_{L}^{n+1}\right)\left(u_{K}^{n+1}-1\right)^{+} \geq 0$. Next, let us establish

$$
G\left(u_{K}^{n+1}, u_{L}^{n+1} ; \eta_{\sigma \sigma^{*}}^{\mathcal{D}} \delta_{L^{*} K^{*}}^{n+1} \xi(u)\right)\left(u_{K}^{n+1}-1\right)^{+} \geq 0 .
$$

So, if $u_{K}^{n+1} \leq 1$ then $\left(u_{K}^{n+1}-1\right)^{+}=0$. Otherwise, $u_{K}^{n+1}>1$, we utilize once again the consistency of $G$ and the fact that it is nonincreasing with respect to the second variable. Therefore

$$
\begin{aligned}
G\left(u_{K}^{n+1}, u_{L}^{n+1} ; \eta_{\sigma \sigma^{*}}^{\mathcal{D}} \delta_{L^{*} K^{*}}^{n+1} \xi(u)\right)\left(u_{K}^{n+1}-1\right)^{+} & \geq G\left(u_{K}^{n+1}, u_{K}^{n+1} ; \eta_{\sigma \sigma^{*}}^{\mathcal{D}} \delta_{L^{*} K^{*}}^{n+1} \xi(u)\right)\left(u_{K}^{n+1}-1\right)^{+} \\
& =-v\left(u_{K}^{n+1}\right) \eta_{\sigma \sigma^{*}}^{\mathcal{D}} \delta_{L^{*} K^{*}}^{n+1} \xi(u)\left(u_{K}^{n+1}-1\right)^{+}=0 .
\end{aligned}
$$

Let us show that $m_{K} \mathcal{P}_{K} u_{\mathcal{T}}\left(u_{K}^{n+1}-1\right)^{+} \geq 0$. We first observe that

$$
m_{K} \mathcal{P}_{K} u_{\mathcal{T}}^{n+1}\left(u_{K}^{n+1}-1\right)^{+}=\frac{1}{h_{\mathfrak{D}}^{\varepsilon}} \sum_{K^{*} \in \overline{\mathfrak{M}^{*}}} m_{K \cap K^{*}}\left(F\left(u_{K}^{n+1}\right)-F(1)+F(1)-F\left(u_{K^{*}}^{n+1}\right)\right)\left(u_{K}^{n+1}-1\right)^{+} .
$$


On the one hand, $\left(F\left(u_{K}^{n+1}\right)-F(1)\right)\left(u_{K}^{n+1}-1\right)^{+}=0$ for every $u_{K}^{n+1} \geq 0$ since $F$ is extended by $F(1)$ on the interval $(1,+\infty)$. On the other hand, $F(1)-F\left(u_{K^{*}}^{n+1}\right) \geq 0$ for all $u_{K^{*}}^{n+1} \in \mathbb{R}$. Thus, $m_{K} \mathcal{P}_{K} u_{\mathcal{T}}\left(u_{K}^{n+1}-1\right)^{+} \geq 0$. Utilizing now the identity

$$
\left(u_{K}^{n+1}-u_{K}^{n}\right)\left(u_{K}^{n+1}-1\right)^{+}=\left(u_{K}^{n+1}-1\right)^{+^{2}}+\left(u_{K}^{n+1}-1\right)^{+}\left(1-u_{K}^{n}\right),
$$

we deduce that $\left(u_{K}^{n+1}-1\right)^{+}=0$, which yields $u_{K}^{n+1} \leq 1$.

Similarly, we mimic the same steps so that we prove the property in the case of the dual mesh. Hence, the proof of the Lemma is concluded.

Remark 4.1. The degeneracy of the function $v$ and the flux splitting scheme (3.4) enforce the boundedness of the discrete solution. Also, this particular approach ensures the coercivity of the discrete elliptic operator.

In the sequel, we will denote by $C$ different constants in various occurrences, which depend only on the physical data together with the regularity of the mesh and are independent of the discretization parameters $\delta t, h_{\mathfrak{D}}$.

\subsection{Estimates on the discrete gradients}

We first recall the following remarkable formula.

Lemma 4.2. (Discrete integration by parts) Let $\mathcal{M}$ be a primal or dual mesh of the domain $\Omega$. For every $K \in \mathcal{M}$, we denote by $N(K)$ the set of neighbors of $K$. Let $A_{K L}, K \in \mathcal{M}$ and $L \in N(K)$ be a real value with $A_{K L}=-A_{L K}$, and let $\varphi$ be a piecewise constant function on the cells of $\mathcal{M}$. Then

$$
\sum_{K \in \mathcal{M}} \sum_{L \in N(K)} A_{K L} \varphi_{K}=-\frac{1}{2} \sum_{K \in \mathcal{M}} \sum_{L \in N(K)} A_{K L}\left(\varphi_{L}-\varphi_{K}\right) .
$$

Particularly, if $A_{K L}=T_{K L}\left(c_{L}-c_{K}\right)$, with $T_{K L}=T_{L K}$, one infers

$$
\sum_{K \in \mathcal{M}} \sum_{L \in N(K)} T_{K L}\left(c_{L}-c_{K}\right) \varphi_{K}=-\frac{1}{2} \sum_{K \in \mathcal{M}} \sum_{L \in N(K)} T_{K L}\left(c_{L}-c_{K}\right)\left(\varphi_{L}-\varphi_{K}\right) .
$$

Proof. The proof of this lemma is omitted since it is similar to that given in [10].

We next refer to $[11,31]$ for the proof of the following fundamental inequality.

Lemma 4.3. (Discrete Poincaré inequality) Consider $\mathcal{T}$ a mesh of $\Omega$. Then there exists a constant $C_{p}$, only depending on the diameter of $\Omega$, such that for every $w_{h} \in X_{\mathcal{T}}$ one has

$$
\left|w_{h}\right|_{2, \mathcal{T}} \leq\left\|w_{\mathfrak{M}}\right\|_{L^{2}(\Omega)}+\left\|w_{\overline{\mathfrak{M}^{*}}}\right\|_{L^{2}(\Omega)} \leq C_{p}\left\|\nabla^{\mathfrak{D}} w_{h}\right\|_{2} .
$$

Proposition 4.1. (Discrete gradient estimate) Let $\left(u_{h}^{n}\right)$, for $n=0, \ldots, N$, be a sequence such that the DDFV scheme (3.10)-(3.12) holds. Then

$$
\sum_{n=0}^{N-1} \delta t\left\|\nabla^{\mathfrak{D}} \xi_{h}^{n+1}\right\|_{2}^{2}+\frac{\gamma}{h_{\mathfrak{D}}^{\varepsilon}} \sum_{n=0}^{N-1} \delta t\left\|\xi\left(u_{\mathfrak{M}}^{n+1}\right)-\xi\left(u_{\mathfrak{M}^{*}}^{n+1}\right)\right\|_{L^{2}(\Omega)}^{2} \leq C,
$$

for some appropriate positive constant $C$.

Proof. We multiply the first (resp. second) equation of the DDFV scheme (3.11)-(3.12) by $u_{K}^{n+1}$ (resp. $u_{K^{*}}^{n+1}$ ) and sum up over all the primal (resp. dual) cells and the integers $n$. Adding together the resulting equations leads to

$$
T_{1}+T_{2}+T_{3}=0
$$


where we have set

$$
\begin{aligned}
T_{1}= & \sum_{n=0}^{N-1} \sum_{K \in \mathfrak{M}} m_{K}\left(u_{K}^{n+1}-u_{K}^{n}\right) u_{K}^{n+1}+\sum_{n=0}^{N-1} \sum_{K^{*} \in \overline{\mathfrak{M}^{*}}} m_{K^{*}}\left(u_{K^{*}}^{n+1}-u_{K^{*}}^{n}\right) u_{K^{*}}^{n+1}, \\
T_{2}= & \sum_{n=0}^{N-1} \delta t \sum_{K \in \mathfrak{M} \mathcal{D}_{\sigma, \sigma^{*}} \in \mathcal{D}_{K}}\left(a_{K L}\left(F_{K}^{n+1}-F_{L}^{n+1}\right)+v_{K L}^{n+1} \eta_{\sigma \sigma^{*}}^{\mathcal{D}}\left(\xi_{K^{*}}^{n+1}-\xi_{L^{*}}^{n+1}\right)\right) u_{K}^{n+1} \\
& +\sum_{n=0}^{N-1} \delta t \sum_{K^{*} \in \overline{\mathfrak{M} *} \mathcal{D}_{\sigma, \sigma^{*} \in \mathcal{D}_{K^{*}}}}\left(a_{K^{*} L^{*}}\left(F_{K^{*}}^{n+1}-F_{L^{*}}^{n+1}\right)+v_{K^{*} L^{*}}^{n+1} \eta_{\sigma \sigma^{*}}^{\mathcal{D}}\left(\xi_{K}^{n+1}-\xi_{L}^{n+1}\right)\right) u_{K^{*}}^{n+1}, \\
T_{3}= & 2 \sum_{n=0}^{N-1} \delta t \gamma \llbracket \mathcal{P} u_{\mathcal{T}}^{n+1}, u_{\mathcal{T}}^{n+1} \rrbracket_{\mathcal{T}} .
\end{aligned}
$$

First of all, observe that

$$
x(x-y) \geq \frac{1}{2}\left(x^{2}-y^{2}\right), \quad \forall x, y \in \mathbb{R} .
$$

According to the above inequality, one can underestimate $T_{1}$

$$
\frac{1}{2} \sum_{K \in \mathfrak{M}} m_{K}\left(\left(u_{K}^{N}\right)^{2}-\left(u_{K}^{0}\right)^{2}\right)+\frac{1}{2} \sum_{K^{*} \in \overline{\mathfrak{M}^{*}}} m_{K^{*}}\left(\left(u_{K^{*}}^{N}\right)^{2}-\left(u_{K^{*}}^{0}\right)^{2}\right) \leq T_{1} .
$$

Let us now turn our attention to the term $T_{2}$. To this end, we perform a discrete integration by parts as given in Lemma 4.2 to obtain

$$
T_{2}=T_{21}+T_{22}
$$

with

$$
\begin{aligned}
T_{21} & =\sum_{n=0}^{N-1} \delta t \sum_{\mathcal{D} \in \mathcal{D}}\left(a_{K L}\left(F_{K}^{n+1}-F_{L}^{n+1}\right)\left(u_{K}^{n+1}-u_{L}^{n+1}\right)+a_{K^{*} L^{*}}\left(F_{K^{*}}^{n+1}-F_{L^{*}}^{n+1}\right)\left(u_{K^{*}}^{n+1}-u_{L^{*}}^{n+1}\right)\right), \\
T_{22} & =\sum_{n=0}^{N-1} \delta t \sum_{\mathcal{D} \in \mathfrak{D}}\left(v_{K L}^{n+1} \eta_{\sigma \sigma^{*}}^{\mathcal{D}}\left(\xi_{K^{*}}^{n+1}-\xi_{L^{*}}^{n+1}\right)\left(u_{K}^{n+1}-u_{L}^{n+1}\right)+v_{K^{*} L^{*}}^{n+1} \eta_{\sigma \sigma^{*}}^{\mathcal{D}}\left(\xi_{K}^{n+1}-\xi_{L}^{n+1}\right)\left(u_{K^{*}}^{n+1}-u_{L^{*}}^{n+1}\right)\right) .
\end{aligned}
$$

The practical inequality (3.8) implies that

$$
T_{21} \geq \sum_{n=0}^{N-1} \delta t \sum_{\mathcal{D} \in \mathcal{D}} a_{K L}\left(\xi_{K}^{n+1}-\xi_{L}^{n+1}\right)^{2}+\sum_{n=0}^{N-1} \delta t \sum_{\mathcal{D} \in \mathcal{D}} a_{K^{*} L^{*}}\left(\xi_{K^{*}}^{n+1}-\xi_{L^{*}}^{n+1}\right)^{2} .
$$

Thanks the monotonicity of the functions $v_{\uparrow}, v_{\downarrow}$ and the definition of $v_{K L}^{n+1}$, we find

$$
v_{K L}^{n+1}\left(u_{K}^{n+1}-u_{L}^{n+1}\right) \eta_{\sigma \sigma^{*}}^{\mathcal{D}}\left(\xi_{K^{*}}^{n+1}-\xi_{L^{*}}^{n+1}\right) \geq \eta_{\sigma \sigma^{*}}^{\mathcal{D}}\left(\xi_{K}^{n+1}-\xi_{L}^{n+1}\right)\left(\xi_{K^{*}}^{n+1}-\xi_{L^{*}}^{n+1}\right) .
$$

Similarly

$$
v_{K^{*} L^{*}}^{n+1}\left(u_{K^{*}}^{n+1}-u_{L^{*}}^{n+1}\right) \eta_{\sigma \sigma^{*}}^{\mathcal{D}}\left(\xi_{K}^{n+1}-\xi_{L}^{n+1}\right) \geq \eta_{\sigma \sigma^{*}}^{\mathcal{D}}\left(\xi_{K}^{n+1}-\xi_{L}^{n+1}\right)\left(\xi_{K^{*}}^{n+1}-\xi_{L^{*}}^{n+1}\right) .
$$

As a result we get

$$
T_{22} \geq 2 \sum_{n=0}^{N-1} \delta t \sum_{\mathcal{D} \in \mathfrak{D}} \eta_{\sigma \sigma^{*}}^{\mathcal{D}}\left(\xi_{K}^{n+1}-\xi_{L}^{n+1}\right)\left(\xi_{K^{*}}^{n+1}-\xi_{L^{*}}^{n+1}\right)
$$


We deduce that

$$
T_{2} \geq \sum_{n=0}^{N-1} \delta t\left(\nabla^{\mathfrak{D}} \xi_{\mathcal{T}}^{n+1}, \nabla^{\mathfrak{D}} \xi_{\mathcal{T}}^{n+1}\right)_{\mathfrak{D}, \Lambda}
$$

In view of the relationship (2.1) and Lemma A.1 we assert

$$
T_{2} \geq C \sum_{n=0}^{N-1} \delta t\left\|\nabla^{\mathcal{D}} \xi_{h}^{n+1}\right\|_{2}^{2},
$$

for some constant $C>0$. Next, owing to the inequality (3.8), we claim

$$
\begin{aligned}
T_{3} & =2 \sum_{n=0}^{N-1} \delta t \gamma \llbracket \mathcal{P} u_{\mathcal{T}}^{n+1}, u_{\mathcal{T}}^{n+1} \rrbracket_{\mathcal{T}} \\
& =\frac{\gamma}{h_{\mathfrak{D}}^{\varepsilon}} \sum_{n=0}^{N-1} \delta t \sum_{K^{*} \in \overline{\mathfrak{M}^{*}}} \sum_{K \in \mathfrak{M}} m_{K \cap K^{*}}\left(F\left(u_{K}^{n+1}\right)-F\left(u_{K^{*}}^{n+1}\right)\right)\left(u_{K}^{n+1}-u_{K^{*}}^{n+1}\right) \\
& \geq \frac{\gamma}{h_{\mathfrak{D}}^{\varepsilon}} \sum_{n=0}^{N-1} \delta t\left\|\xi\left(u_{\mathfrak{M}^{n+1}}^{n+1}\right)-\xi\left(u_{\mathfrak{M}^{*}}^{n+1}\right)\right\|_{L^{2}(\Omega)}^{2} .
\end{aligned}
$$

Also, observe that

$$
T_{2}+T_{3}=-T_{1} \leq\left\|u^{0}\right\|_{L^{2}(\Omega)}^{2} .
$$

Finally, combining(4.3)-(4.5), the energy estimate (4.2) follows as required.

Corollary 4.1. From the previous proposition, one gets

$$
\sum_{n=0}^{N-1} \delta t\left\|\nabla^{\mathfrak{D}} F_{h}^{n+1}\right\|_{2}^{2} \leq C
$$

Proof. This result is a direct consequence of Lemma A.1 together with inequality (4.2). It is sufficient to observe that

$$
F(a)-F(b)=v\left(x_{0}\right)(\xi(a)-\xi(b))
$$

for some $x_{0} \in[\min (a, b), \max (a, b)]$ and notice that the function $v$ is bounded.

\section{Existence of discrete solutions}

In this section, we prove that the nonlinear algebraic system, which comes from the DDFV scheme, admits a solution. To this end, we will need the following fundamental lemma, that can be found in [29]. This result ensures the existence of at least one zero to some specific vector fields.

Lemma 5.1. Let $\mathcal{A}$ be a finite dimensional Hilbert space with inner product $(\cdot, \cdot)$ and norm $\|\cdot\|$, and let $\mathcal{L}$ be a continuous mapping from $\mathcal{A}$ into itself which verifies

$$
(\mathcal{L}(x), x)>0, \text { for }\|x\|=r>0 .
$$

Then, there exists $x^{*} \in \mathcal{A}$ with $\left\|x^{*}\right\|<r$ such that

$$
\mathcal{L}\left(x^{*}\right)=0 .
$$

We now state the existence result in the proposition below.

Proposition 5.1. The DDFV scheme (3.10)-(3.12) has at least one solution $u_{\mathcal{T}}^{n+1}$ for every $n=0, \ldots, N-$ 1. 
Proof. We proceed by induction on $n$. We then assume that $u_{\mathcal{T}}^{n}$ is given and prove the existence of $u_{\mathcal{T}}^{n+1}$ satisfying the numerical scheme (3.11)-(3.12). To this purpose, we define the mapping $\mathcal{L}: \mathbb{R}^{\# \mathcal{T}} \longrightarrow \mathbb{R}^{\# \mathcal{T}}$ that associates for each $u_{\mathcal{T}}^{n+1}$ the vector :

$$
\mathcal{L}\left(u_{\mathcal{T}}^{n+1}\right)=\left(\mathcal{L}_{M}\right)_{M \in \mathcal{T}}
$$

where

$$
\begin{aligned}
& \mathcal{L}_{K}=\frac{m_{K}}{\delta t}\left(u_{K}^{n+1}-u_{K}^{n}\right) \\
& +\sum_{\mathcal{D}_{\sigma, \sigma^{*}} \in \mathcal{D}_{K}}\left(a_{K L}\left(F_{K}^{n+1}-F_{L}^{n+1}\right)+v_{K L}^{n+1} \eta_{\sigma \sigma^{*}}^{\mathcal{D}}\left(\xi_{K^{*}}^{n+1}-\xi_{L^{*}}^{n+1}\right)\right)+\gamma m_{K} \mathcal{P}_{K} u_{\mathcal{T}}^{n+1}, \text { if } M=K \in \mathfrak{M}, \\
& \mathcal{L}_{K^{*}}=\frac{m_{K^{*}}}{\delta t}\left(u_{K^{*}}^{n+1}-u_{K^{*}}^{n}\right) \\
& +\sum_{\mathcal{D}_{\sigma, \sigma^{*}} \in \mathcal{D}_{K^{*}}}\left(a_{K^{*} L^{*}}\left(F_{K^{*}}^{n+1}-F_{L^{*}}^{n+1}\right)+v_{K^{*} L^{*}}^{n+1} \eta_{\sigma \sigma^{*}}^{\mathcal{D}}\left(\xi_{K}^{n+1}-\xi_{L}^{n+1}\right)\right)+\gamma m_{K^{*}} \mathcal{P}_{K^{*}} u_{\mathcal{T}}^{n+1}, \text { if } M=K^{*} \in \mathfrak{M}^{*}, \\
& \mathcal{L}_{K^{*}}=0, \text { if } M=K^{*} \in \partial \mathfrak{M}^{*} .
\end{aligned}
$$

The functional $\mathcal{L}$ is well-defined and continuous. It remains to demonstrate that

$$
\left(\mathcal{L}\left(u_{\mathcal{T}}^{n+1}\right), u_{\mathcal{T}}^{n+1}\right)>0, \text { for }\left\|u_{\mathcal{T}}^{n+1}\right\|_{\mathbb{R}^{\# \mathcal{T}}}=r
$$

for some sufficiently large $r$. It follows from the calculation of the previous section, Lemma 4.1, stating that $0 \leq u_{K}^{n}, u_{K^{*}}^{n} \leq 1$ for all $K, K^{*}$, and the Poincaré inequality given in Lemma 4.3 that

$$
\begin{aligned}
\left(\mathcal{L}\left(u_{\mathcal{T}}^{n+1}\right), u_{\mathcal{T}}^{n+1}\right) & \geq \frac{1}{\delta t} \sum_{K \in \mathfrak{M}} m_{K}\left(\left(u_{K}^{n+1}\right)^{2}-\left(u_{K}^{n}\right)^{2}\right)+\frac{1}{\delta t} \sum_{K^{*} \in \overline{M^{*}}} m_{K^{*}}\left(\left(u_{K^{*}}^{n+1}\right)^{2}-\left(u_{K^{*}}^{n}\right)^{2}\right) \\
& +C\left\|\nabla^{\mathcal{D}} \xi_{\mathcal{T}}^{n+1}\right\|_{2}^{2} \\
& \geq C^{\prime}\left|u_{\mathcal{T}}^{n+1}\right|_{2, \mathcal{T}}^{2}-\frac{2|\Omega|}{\delta t},
\end{aligned}
$$

for some constants $C, C^{\prime}>0$. Thanks to the equivalence of the usual norms $\|\cdot\|_{\mathbb{R}^{\#} \mathcal{T}},|\cdot|_{2, \mathcal{T}}$ on the finite dimensional space $\mathbb{R}^{\# \mathcal{T}}$, inequality (5.1) is fulfilled provided a large $r$. We therefore obtain the existence of at least one solution to the nonlinear DDFV scheme (3.10)-(3.12).

\section{Convergence}

We first give some standard compactness properties. Their proofs follow similar arguments as, for instance, in $[6,31]$.

Lemma 6.1. (Space translates)

Let $u_{h, \delta t}$ be a discrete solution to the DDFV scheme (3.10)-(3.12). Then

$$
\int_{0}^{T} \int_{\Omega^{\prime}}\left|\xi_{h, \delta t}(x+y, t)-\xi_{h, \delta t}(x, t)\right| \mathrm{d} x \mathrm{~d} t \leq \omega(|y|), \text { for every } y \in \mathbb{R}^{2},
$$

where $\Omega^{\prime}=\{x \in \Omega / x+y \in \Omega\}$ and $\omega$ is a modulus of continuity independent of $\delta t, h_{\mathfrak{D}}$, verifying $\omega(|y|) \longrightarrow 0$ as $|y| \longrightarrow 0$.

Lemma 6.2. (Time translates)

Let $u_{h, \delta t}$ be a solution to the DDFV scheme (3.10)-(3.12). Then there exists a constant $C$ that does not depend on $h_{\mathfrak{D}}$ nor on $\delta t$ such that

$$
\begin{aligned}
& \int_{0}^{T-\tau} \int_{\Omega}\left|\xi\left(u_{\mathfrak{M}, \delta t}(x, t+\tau)\right)-\xi\left(u_{\mathfrak{M}, \delta t}(x, t)\right)\right|^{2} \mathrm{~d} x \mathrm{~d} t \\
& +\int_{0}^{T-\tau} \int_{\Omega}\left|\xi\left(u_{\overline{\mathfrak{M}^{*}}, \delta t}(x, t+\tau)\right)-\xi\left(u_{\overline{\mathfrak{M}^{*}}, \delta t}(x, t)\right)\right|^{2} \mathrm{~d} x \mathrm{~d} t \leq C \tau,
\end{aligned}
$$


for all $\tau \in(0, T)$.

We now claim a weak convergence of the discrete gradient and a strong convergence of $u_{h, \delta t}$.

Proposition 6.1. Let $\mathcal{T}_{h}$ be a sequence of DDFV meshes such that $h_{\mathfrak{D}}, \delta$ t tend to zero and $\operatorname{reg}\left(\mathcal{T}_{h}\right)$ is bounded. Then, the following convergences hold up to a subsequence:

$$
\begin{aligned}
& u_{h, \delta t}, u_{\mathfrak{M}_{h}, \delta t}, u_{\overline{\mathfrak{M}^{*}}, \delta t} \longrightarrow u \quad \text { a.e. in } Q_{T}, \\
& \nabla^{\mathcal{D}} F_{h, \delta t} \longrightarrow \nabla F(u) \quad \text { weakly } \quad \text { in } L^{2}\left(Q_{T}\right)^{2} \text {. }
\end{aligned}
$$

Moreover

$$
0 \leq u \leq 1 \quad \text { a.e. in } Q_{T} .
$$

Proof. The space and time translate estimations are the main ingredients that allow us to apply a discrete version [31] of Kolmogorov's compactness theorem [13], claiming that the sequences $\xi\left(u_{\mathfrak{M}_{h}, \delta t}\right), \xi\left(u_{\overline{\mathfrak{M}}^{*}}, \delta t\right)$ are relatively compact in $L^{1}\left(Q_{T}\right)$. This ensures the existence of unlabeled subsequences still denoted

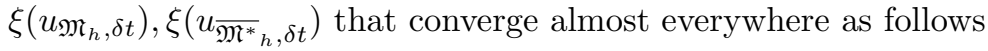

$$
\xi\left(u_{\mathfrak{M}_{h}, \delta t}\right) \longrightarrow \xi_{1} \text { a.e. in } Q_{T} \text {, and } \xi\left(u_{\overline{\mathfrak{M}}^{*}}, \delta t\right) \longrightarrow \xi_{2} \text { a.e. in } Q_{T},
$$

for some functions $\xi_{1}$ and $\xi_{2}$. Since $\xi^{-1}$ is continuous, we deduce that

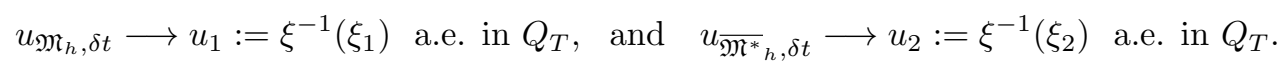

In light of Proposition 4.1, we assert

$$
\left\|\xi\left(u_{\mathfrak{M}, \delta t}\right)-\xi\left(u_{\overline{\mathfrak{M}^{*}}, \delta t}\right)\right\|_{L^{2}\left(Q_{T}\right)}^{2} \leq C h_{\mathfrak{D}}^{\varepsilon} .
$$

Thus, up to another unlabeled subsequence, we get

$$
\xi\left(u_{\mathfrak{M}, \delta t}\right)-\xi\left(u_{\overline{\mathfrak{M}^{*}}, \delta t}\right) \longrightarrow 0, \text { a.e. in } Q_{T} .
$$

Therefore

$$
u_{\mathfrak{M}, \delta t}-u_{\overline{\mathfrak{M}^{*}, \delta t}} \longrightarrow 0 \text {, a.e. in } Q_{T} .
$$

We then verify that $u_{1}=u_{2}:=u$. Consequently

$$
u_{h, \delta t} \longrightarrow u \text { a.e. in } Q_{T} \text {, and } \xi_{h, \delta t} \longrightarrow \xi(u) \text { a.e. in } Q_{T} \text {. }
$$

Thanks to the $L^{\infty}$ bound given in Lemma 4.1, we deduce from Lebesgue's dominated convergence theorem that

$$
\lim _{h_{\mathfrak{D}}, \delta t \rightarrow 0}\left\|u_{h, \delta t}-u\right\|_{L^{2}\left(Q_{T}\right)}=0 .
$$

Thereby

$$
\lim _{h_{\mathfrak{D}, \delta t \rightarrow 0}}\left\|F_{h, \delta t}-F(u)\right\|_{L^{2}\left(Q_{T}\right)}=0 .
$$

Next, thanks to Corollary 4.1 , the sequence $\left(\nabla^{\mathfrak{D}} F_{h, \delta t}\right)$ is bounded in $\left(L^{2}\left(Q_{T}\right)\right)^{2}$. Following [6] we establish that

$$
\nabla^{\mathfrak{D}} F_{h, \delta t} \longrightarrow \nabla F(u) \quad \text { weakly in }\left(L^{2}\left(Q_{T}\right)\right)^{2} .
$$




\section{Passage to the limit}

In this section we prove that any limit of the approximate solution sequence converges towards the weak solution of the main problem.

Theorem 7.1. Under hypotheses $\left(A_{1}\right)-\left(A_{3}\right)$ and assuming a uniform boundedness of the mesh regularity, the limit function $u$ of Proposition 6.1 is the weak solution to the problem (1.1) in the sense of Definition 1.1 .

Proof. Let $\psi \in \mathcal{C}_{c}^{\infty}(\Omega \times[0, T))$, we denote by $\psi_{K}^{n+1}=\psi\left(x_{K}, t^{n+1}\right)$ and $\psi_{K^{*}}^{n+1}=\psi\left(x_{K^{*}}, t^{n+1}\right)$. We multiply the equations (3.11), (3.12) by $\frac{1}{2} \delta t \psi_{K}^{n+1}, \frac{1}{2} \delta t \psi_{K^{*}}^{n+1}$ respectively, sum over $K, K^{*}$ and $n$. Next, one performs an integration by parts, adds and substracts $\sum_{n=0}^{N-1} \delta t\left(\nabla^{\mathfrak{D}} F_{\mathcal{T}_{h}}^{n+1}, \nabla^{\mathfrak{D}} \psi_{\mathcal{T}_{h}}^{n+1}\right)_{\mathfrak{D}, \Lambda}$ to get

$$
\mathcal{S}_{\mathcal{T}_{h}, \delta t}^{1}+\mathcal{S}_{\mathcal{T}_{h}, \delta t}^{2}+\mathcal{S}_{\mathcal{T}_{h}, \delta t}^{3}+\mathcal{S}_{\mathcal{T}_{h}, \delta t}^{4}=0
$$

where

$$
\begin{aligned}
\mathcal{S}_{\mathcal{T}_{h}, \delta t}^{1}= & \sum_{n=0}^{N-1} \llbracket u_{\mathcal{T}_{h}}^{n+1}-u_{\mathcal{T}_{h}}^{n}, \psi_{\mathcal{T}_{h}}^{n+1} \rrbracket_{\mathcal{T}_{h}}, \\
\mathcal{S}_{\mathcal{T}_{h}, \delta t}^{2}= & \sum_{n=0}^{N-1} \delta t\left(\nabla^{\mathcal{D}} F_{\mathcal{T}_{h}}^{n+1}, \nabla^{\mathcal{D}} \psi_{\mathcal{T}_{h}}^{n+1}\right)_{\mathcal{D}, \Lambda}=\int_{Q_{T}} \Lambda \nabla^{\mathcal{D}} F_{\mathcal{T}_{h}}^{n+1} \cdot \nabla^{\mathcal{D}} \psi_{\mathcal{T}_{h}}^{n+1} \mathrm{~d} x \mathrm{~d} t, \\
\mathcal{S}_{\mathcal{T}_{h}, \delta t}^{3}= & \frac{1}{2} \sum_{n=0}^{N-1} \delta t \sum_{\mathcal{D} \in \mathcal{D}_{h}} \eta_{\sigma \sigma^{*}}^{\mathcal{D}}\left[v_{K L}^{n+1}\left(\xi_{K^{*}}^{n+1}-\xi_{L^{*}}^{n+1}\right)-\left(F_{K^{*}}^{n+1}-F_{L^{*}}^{n+1}\right)\right]\left(\psi_{K}^{n+1}-\psi_{L}^{n+1}\right) \\
& \quad+\frac{1}{2} \sum_{n=0}^{N-1} \delta t \sum_{\mathcal{D} \in \mathfrak{D}_{h}} \eta_{\sigma \sigma^{*}}^{\mathcal{D}}\left[v_{K^{*} L^{*}}^{n+1}\left(\xi_{K}^{n+1}-\xi_{L}^{n+1}\right)-\left(F_{K}^{n+1}-F_{L}^{n+1}\right)\right]\left(\psi_{K^{*}}^{n+1}-\psi_{L^{*}}^{n+1}\right), \\
\mathcal{S}_{\mathcal{T}_{h}, \delta t}^{4}= & \gamma \sum_{n=0}^{N-1} \delta t \llbracket \mathcal{P}_{\mathcal{T}_{h}}^{n+1}, \psi_{\mathcal{T}_{h}}^{n+1} \rrbracket_{\mathcal{T}_{h}} .
\end{aligned}
$$

Let us start off by establishing

$$
\lim _{h_{\mathfrak{D}}, \delta t \rightarrow 0} \mathcal{S}_{\mathcal{T}_{h}, \delta t}^{1}=-\int_{\Omega} u^{0} \psi(\cdot, 0) \mathrm{d} x-\int_{Q_{T}} u \partial_{t} \psi \mathrm{d} x \mathrm{~d} t .
$$

Using a summation by parts in time and the fact that $\psi_{K}^{N}=\psi_{K^{*}}^{N}=0$, yields

$$
\begin{aligned}
\mathcal{S}_{\mathcal{T}_{h}, \delta t}^{1} & =-\llbracket u_{\mathcal{T}_{h}}^{0}, \psi_{\mathcal{T}_{h}}(\cdot, 0) \rrbracket \mathcal{T}_{h}-\sum_{n=0}^{N-1} \llbracket u_{\mathcal{T}_{h}}^{n}, \psi_{\mathcal{T}_{h}}^{n+1}-\psi_{\mathcal{T}_{h}}^{n} \rrbracket \mathcal{T}_{h} \\
& =: \mathcal{S}_{\mathcal{T}_{h}, \delta t}^{1,1}+\mathcal{S}_{\mathcal{T}_{h}, \delta t}^{1,2} .
\end{aligned}
$$

Thanks to the strong convergence of $\left(\psi_{\mathcal{T}_{h}}(\cdot, 0)\right)$, one obtains

$$
\lim _{h_{\mathcal{D}}, \delta t \rightarrow 0} \mathcal{S}_{\mathcal{T}_{h}, \delta t}^{1,1}=-\int_{\Omega} u^{0} \psi(\cdot, 0) \mathrm{d} x .
$$

Expanding the term $\mathcal{S}_{\mathcal{T}_{h}, \delta t}^{1,2}$ entails

$$
\begin{aligned}
\mathcal{S}_{\mathcal{T}_{h}, \delta t}^{1,2} & =-\sum_{n=0}^{N-1} \llbracket u_{\mathcal{T}_{h}}^{n}, \psi_{\mathcal{T}_{h}}^{n+1}-\psi_{\mathcal{T}_{h}}^{n} \rrbracket \rrbracket_{\mathcal{T}_{h}} \\
& =-\frac{1}{2} \sum_{n=0}^{N-1} \sum_{K \in \mathfrak{M}} m_{K} \int_{t^{n}}^{t^{n+1}} u_{K}^{n} \partial_{t} \psi\left(x_{K}, t\right) \mathrm{d} x \mathrm{~d} t-\frac{1}{2} \sum_{n=0}^{N-1} \sum_{K^{*} \in \overline{\mathfrak{M}^{*}}} m_{K^{*}} \int_{t^{n}}^{t^{n+1}} u_{K^{*}}^{n} \partial_{t} \psi\left(x_{K^{*}}, t\right) \mathrm{d} x \mathrm{~d} t .
\end{aligned}
$$


Bearing in mind that $\left(\partial_{t} \psi\left(x_{K}, \cdot\right)\right)_{K \in \mathfrak{M}}$ and $\left(\partial_{t} \psi\left(x_{K^{*}}, \cdot\right)\right)_{K^{*} \in \overline{\mathfrak{M}^{*}}}$ converge uniformly towards $\partial_{t} \psi$, we apply (6.3) together with the Lebesgue dominated convergence theorem to find

$$
\lim _{h_{\mathfrak{D}}, \delta t \rightarrow 0} \mathcal{S}_{\mathcal{T}_{h}, \delta t}^{1,2}=\lim _{h_{\mathfrak{D}}, \delta t \rightarrow 0} \mathcal{S}_{\mathcal{T}_{h}, \delta t}^{1,2}=-\int_{Q_{T}} u \partial_{t} \psi \mathrm{d} x \mathrm{~d} t .
$$

Let us next prove the convergence of the diffusion part. To do so, we recall that the sequence $\left(\nabla^{\mathfrak{D}} F_{\mathcal{T}_{h}}^{n+1}\right)$ converges weakly towards $\nabla F(u)$ whereas $\left(\Lambda \nabla^{\mathfrak{D}} \psi_{\mathcal{T}_{h}}^{n+1}\right)$ converges uniformly towards $\Lambda \nabla \psi$. Thereby

$$
\lim _{h_{\mathfrak{D}}, \delta t \rightarrow 0} \mathcal{S}_{\mathcal{T}_{h}, \delta t}^{2}=\int_{Q_{T}} \Lambda \nabla F(u) \cdot \nabla \psi \mathrm{d} x \mathrm{~d} t .
$$

Let us turn our attention to the convergence of $\mathcal{S}_{\mathcal{T}_{h}, \delta t}^{3}$. This term can be split up into two parts as follows

$$
\mathcal{S}_{\mathcal{T}_{h}, \delta t}^{3}=\mathcal{S}_{\mathcal{T}_{h}, \delta t}^{3,1}+\mathcal{S}_{\mathcal{T}_{h}, \delta t}^{3,2},
$$

where we have set

$$
\begin{aligned}
& \mathcal{S}_{\mathcal{T}_{h}, \delta t}^{3,1}=\frac{1}{2} \sum_{n=0}^{N-1} \delta t \sum_{\mathcal{D} \in \mathfrak{D}_{h}} \eta_{\sigma \sigma^{*}}^{\mathcal{D}}\left[v_{K L}^{n+1}\left(\xi_{K^{*}}^{n+1}-\xi_{L^{*}}^{n+1}\right)-\left(F_{K^{*}}^{n+1}-F_{L^{*}}^{n+1}\right)\right]\left(\psi_{K}^{n+1}-\psi_{L}^{n+1}\right), \\
& \mathcal{S}_{\mathcal{T}_{h}, \delta t}^{3,2}=\frac{1}{2} \sum_{n=0}^{N-1} \delta t \sum_{\mathcal{D} \in \mathfrak{D}_{h}} \eta_{\sigma \sigma^{*}}^{\mathcal{D}}\left[v_{K^{*} L^{*}}^{n+1}\left(\xi_{K}^{n+1}-\xi_{L}^{n+1}\right)-\left(F_{K}^{n+1}-F_{L}^{n+1}\right)\right]\left(\psi_{K^{*}}^{n+1}-\psi_{L^{*}}^{n+1}\right) .
\end{aligned}
$$

Next, the first mean value theorem guarantees the existence of a constant

$$
u_{K^{*} L^{*}} \in\left[\min \left(u_{K^{*}}^{n+1}, u_{L^{*}}^{n+1}\right), \max \left(u_{K^{*}}^{n+1}, u_{L^{*}}^{n+1}\right)\right]
$$

satisfying

$$
F_{K^{*}}^{n+1}-F_{L^{*}}^{n+1}=v\left(u_{K^{*} L^{*}}\right)\left(\xi_{K^{*}}^{n+1}-\xi_{L^{*}}^{n+1}\right) .
$$

Thus, using Assumption $\left(A_{3}\right)$ on the tensor $\Lambda$ and the regularity of the mesh, we get

$$
\left|\mathcal{S}_{\mathcal{T}_{h}, \delta t}^{3,1}\right| \leq C \sum_{n=0}^{N-1} \delta t \sum_{\mathcal{D} \in \mathfrak{D}_{h}} m_{\mathcal{D}}\left|v_{K L}^{n+1}-v\left(u_{K^{*} L^{*}}\right)\right|\left|\nabla^{\mathcal{D}} \xi_{\mathcal{T}, \delta t}\right|\left|\nabla^{\mathcal{D}} \psi_{\mathcal{T}_{h}, \delta t}\right| .
$$

for some constant $C>0$. We set

$$
\begin{array}{ll}
\bar{\xi}_{\mathcal{D}}^{n+1}:=\max _{M \in \mathcal{V}_{\mathcal{D}}}\left\{\xi\left(u_{M}^{n+1}\right)\right\}, & \underline{\xi}_{\mathcal{D}}^{n+1}:=\min _{M \in \mathcal{V}_{\mathcal{D}}}\left\{\xi\left(u_{M}^{n+1}\right)\right\} \\
\bar{\xi}_{\mathcal{T}, \delta t \mid \mathcal{D} \times\left(t^{n}, t^{n+1}\right]}:=\bar{\xi}_{\mathcal{D}}^{n+1}, & \underline{\xi}_{\mathcal{T}, \delta t} \mid \mathcal{D} \times\left(t^{n}, t^{n+1}\right]:=\underline{\xi}_{\mathcal{D}}^{n+1},
\end{array}
$$

where $\mathcal{V}_{\mathcal{D}}$ stands for the set of vertices of the diamond $\mathcal{D}$. The function $\xi$ is increasing and continuous on $[0,1]$ then its inverse is continuous on the compact $[0, \xi(1)]$. Therefore, there exists a modulus of continuity $\omega$ of $v \circ \xi^{-1}$, which is continuous and bounded on the same interval with $\omega(0)=0$. This latter fact and the Cauchy-Schwarz inequality yields

$$
\begin{aligned}
\left|\mathcal{S}_{\mathcal{T}_{h}, \delta t}^{3,1}\right| & \leq C\|\nabla \psi\|_{\infty} \sum_{n=0}^{N-1} \delta t \sum_{\mathcal{D} \in \mathfrak{D}_{h}} m_{\mathcal{D}} \omega\left(\bar{\xi}_{\mathcal{D}}^{n+1}-\underline{\xi}_{\mathcal{D}}^{n+1}\right)\left|\nabla^{\mathcal{D}} \xi_{\mathcal{T}, \delta t}\right| \\
& \leq C\left(\sum_{n=0}^{N-1} \delta t \sum_{\mathcal{D} \in \mathfrak{D}_{h}} m_{\mathcal{D}} \omega\left(\bar{\xi}_{\mathcal{D}}^{n+1}-\underline{\xi}_{\mathcal{D}}^{n+1}\right)^{2}\right)^{1 / 2} \times\left(\sum_{n=0}^{N-1} \delta t \sum_{\mathcal{D} \in \mathfrak{D}_{h}} m_{\mathcal{D}}\left|\nabla^{\mathcal{D}} \xi_{\mathcal{T}, \delta t}\right|^{2}\right)^{1 / 2} \\
& \leq C\left(\int_{Q_{T}} \omega\left(\bar{\xi}_{\mathcal{T}, \delta t}-\underline{\xi}_{\mathcal{T}, \delta t}\right)^{2} \mathrm{~d} x \mathrm{~d} t\right)^{1 / 2} \times\left(\sum_{n=0}^{N-1} \delta t\left\|\nabla^{\mathcal{D}} \xi_{\mathcal{T}}^{n+1}\right\|_{2}^{2}\right)^{1 / 2}
\end{aligned}
$$


for some positive constant $C$. In view of Lemma A.2 together with (4.2), we deduce that

$$
\lim _{h_{\mathfrak{D}}, \delta t \rightarrow 0} \mathcal{S}_{\mathcal{T}_{h}, \delta t}^{3,1}=0
$$

Similarly, we establish that

$$
\lim _{h_{\mathfrak{D}}, \delta t \rightarrow 0} \mathcal{S}_{\mathcal{T}_{h}, \delta t}^{3,2}=0
$$

Finally, let us demonstrate that

$$
\lim _{h_{\mathfrak{D}}, \delta t \rightarrow 0} \mathcal{S}_{\mathcal{T}_{h}, \delta t}^{4}=0 .
$$

Owing to the definition of the penalization term we explore

$$
\begin{aligned}
& \sum_{n=0}^{N-1} \delta t\left|\llbracket \mathcal{P} u_{\mathcal{T}_{h}}^{n+1}, \psi_{\mathcal{T}_{h}}^{n+1} \rrbracket_{\mathcal{T}_{h}}\right|=\left|\frac{1}{2} \frac{1}{h_{\mathfrak{D}}^{\varepsilon}} \sum_{n=0}^{N-1} \delta t \sum_{K^{*} \in \bar{M}^{*}} \sum_{K \in \mathfrak{M}} m_{K \cap K^{*}}\left(F\left(u_{K}^{n+1}\right)-F\left(u_{K^{*}}^{n+1}\right)\right)\left(\psi_{K}^{n+1}-\psi_{K^{*}}^{n+1}\right)\right| \\
& \leq \frac{1}{2} \frac{\|v\|_{\infty}}{h_{\mathfrak{D}}^{\varepsilon}} \sum_{n=0}^{N-1} \delta t \sum_{K^{*} \in \overline{\mathfrak{M}}^{*}} \sum_{K \in \mathfrak{M}} m_{K \cap K^{*}}\left|\xi_{K}^{n+1}-\xi_{K^{*}}^{n+1}\right|\left|\psi_{K}^{n+1}-\psi_{K^{*}}^{n+1}\right| \\
& \leq \frac{1}{2} \frac{\|v\|_{\infty}}{h_{\mathfrak{D}}^{\varepsilon}}\left\|\xi_{\mathfrak{M}_{h}, \delta t}-\xi_{\overline{\mathfrak{M}^{*}}{ }_{h}, \delta t}\right\|_{L^{2}\left(Q_{T}\right)}\left\|\psi_{\mathfrak{M}_{h}, \delta t}-\psi_{\overline{\mathfrak{M}^{*}}{ }_{h}, \delta t}\right\|_{L^{2}\left(Q_{T}\right)} .
\end{aligned}
$$

On the other hand, the regularity of the function $\psi$ ensures the existence of a constant $C$ depending only on the regularity of the mesh such that (see [20] for deep details)

$$
\left\|\psi_{\mathfrak{M}_{h}, \delta t}-\psi_{\overline{\mathfrak{M}^{*}}, \delta t}\right\|_{L^{2}\left(Q_{T}\right)} \leq C h_{\mathfrak{D}}\|\psi\|_{W^{1, \infty}(\Omega)} .
$$

Utilizing the energy estimate (4.2) and the fact that $\varepsilon<2$ we obtain

$$
\left|\mathcal{S}_{\mathcal{T}_{h}, \delta t}^{4}\right| \leq C h_{\mathfrak{D}}^{1-\varepsilon / 2} \longrightarrow 0, \quad h_{\mathfrak{D}}, \delta t \longrightarrow 0
$$

This ends the proof of the theorem.

\section{Numerical results}

In this section, we present some numerical tests so that we can show the efficiency and the stability of the proposed DDFV scheme. As highlighted in the introduction of this work, this method will allow us to take into account almost general meshes and any tensor. We also stress that boundary conditions of Dirichlet type are prescribed. To take them into consideration, it is sufficient to take the trace of a given exact solution on the boundary. This particularity provides analytical solutions of the continuous problem and enables us to compare them with the discrete ones.

To begin with, let us consider the unit square $\Omega=[0,1]^{2}$ as the domain of our study. Next, the primal meshes are given by a sequence of distorted quadrangulation, Kershaw and triangular meshes of $\Omega$. The first family is denoted by $M 1$ while the second one is denoted by $M 2$. These kinds of meshes are taken from the FVCA5 benchmark [39]. Their corresponding dual meshes are constructed as described in Section 2. The first element of the sequence $M 2$ as well as the third element of the family $M 1$ are depicted on Figure 3. The mobility function is chosen as follows

$$
f(u)=u^{m}(1-u)^{m}, \quad \forall u \in[0,1] \text { and } m \in\{1,2\} .
$$

Notice that this function presents some degeneracy at $u=0$ and at $u=1$. Additionally, we require the computation of the functions $v_{\uparrow}(u)$ and $v_{\downarrow}(u)$ in order to calculate the numerical flux. In our study, the function $v$ admits a unique global maximum $\bar{u}=1 / 2$. Hence, one gets in a straightforward way that

$$
v_{\uparrow}(u)=v\left(\min \left\{u, \frac{1}{2}\right\}\right), \quad \text { and } v_{\downarrow}(u)=v\left(\max \left\{u, \frac{1}{2}\right\}\right)-v\left(\frac{1}{2}\right), \quad \text { for all } u \in(0,1)^{2} .
$$



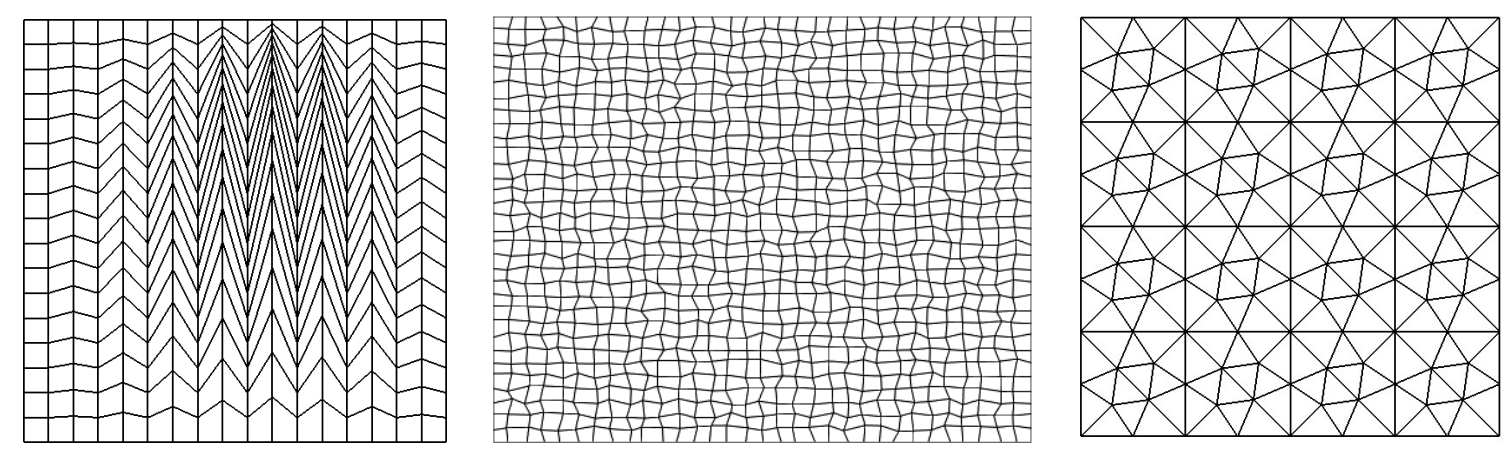

Figure 3: From left to right, Kershaw, quadrangle and triangular meshes.

We also focus on the case of anisotropic media to verify the validity of our discretization. To this end, we select a diagonal tensor $\Lambda$ :

$$
\Lambda=\left(\begin{array}{cc}
\Lambda_{x x} & 0 \\
0 & \Lambda_{y y}
\end{array}\right)
$$

The DDFV scheme is formulated in a nonlinear algebraic system, which is solved thanks to Newton's method with a given tolerance of $10^{-10}$. We underline that the numerical scheme (3.11)-(3.12) is fully implicit in time, unconditionally stable and convergent. Yet, we require the time step to be proportional to the square of the mesh size as mentioned in [19] to assess numerical errors.

As we are interested in the accuracy of scheme, we are going to evaluate the error of the proposed discretization. In all the tests, we denote by ERL2 the difference between the analytical solution and the numerical one in $L^{\infty}\left(0, T ; L^{2}(\Omega)\right)$. Moreover, we study the error between the gradient of the continuous semi-Kirchhoff function $\xi$ and its discrete counterpart in $L^{2}(\Omega \times(0, T))^{2}$, which is denoted by ERGL2. The convergence rate will be designated by Rate. More precisely

$$
\begin{gathered}
E R L \mathscr{2}=\left\|u_{\mathrm{ex}}-u_{h, \delta t}\right\|_{L^{\infty}\left(0, T ; L^{2}(\Omega)\right)}, \quad E R G L 2=\left\|\nabla \xi\left(u_{\mathrm{ex}}\right)-\nabla \xi\left(u_{h, \delta t}\right)\right\|_{L^{2}(\Omega \times(0, T))^{2} .} \\
\text { Rate }=\frac{\log \left(E r r^{i+1} / E r r^{i}\right)}{\log \left(h_{\mathfrak{D}}^{i+1} / h_{\mathfrak{D}}^{i}\right)}, \quad \operatorname{Err}=E R L 2, E R G L 2,
\end{gathered}
$$

where $i$ refers to the index of the space discretization $\mathcal{T}_{i}$ for $i=1, \cdots, 5$. In all the tables below $u_{\text {min }}$ (resp. $u_{\max }$ ) stands for the minimum (resp. maximum) of the computed solution. The parameter $h_{\mathfrak{D}}^{i}$ is the biggest diameter of the diamonds build on $\mathcal{T}_{i}$.

\section{Test 1}

In this test, we investigate the numerical convergence of the DDFV scheme (3.10)-(3.12) using the exact solution:

$$
u_{\mathrm{ex}}(x, t)=80 x_{1}^{2}\left(1-x_{1}\right)^{2} \times t, \quad \forall x=\left(x_{1}, x_{2}\right) \in \Omega, \quad t \in(0, T) .
$$

Substituting this expression in the main problem (1.1) yields a nonnegative source term. One notices that this solution degenerates at the line $\left\{x_{1}=0\right\}$ and at $\left\{x_{1}=1\right\}$. The mobility function $f(u)=u^{2}(1-u)^{2}$ is considered. Here, the final time is fixed to $T=0.15$.

First, we have seen that the penalization term has played a crucial role to establish that the two reconstructions of the solution on the primal and dual meshes converge to the same limit. Second, this fact holds numerically without the penalty term. To see this, we compute the difference in $L^{2}(\Omega \times(0, T))$ norm between the approximate solution on the primal mesh and that on the dual mesh. For this, we consider two values of the stabilization parameter $\gamma=0$ and $\gamma=0.5$ with a fixed $\varepsilon=1$. As shown in Table 1 , the presence or the absence of the penalization term does not influence the convergence of the sequence 


\begin{tabular}{|c|c|c|c|c|c|}
\hline & & \multicolumn{2}{|l|}{$\gamma=0$} & \multicolumn{2}{|l|}{$\gamma=0.5$} \\
\hline$h_{\mathfrak{D}}$ & $\# \mathcal{T}$ & $\left\|u_{\mathfrak{M}, \delta t}-u_{\overline{\mathfrak{M}^{*}, \delta t}}\right\|$ & Rate & $\left\|u_{\mathfrak{M}, \delta t}-u_{\overline{\mathfrak{M}^{*}, \delta t}}\right\|$ & Rate \\
\hline 0.342 & 41 & 0.111 E-01 & - & 0.110 E-01 & - \\
\hline 0.174 & 145 & 0.575 E-02 & 0.974 & 0.574 E-02 & 0.973 \\
\hline 0.092 & 545 & 0.296 E-02 & 1.034 & 0.295 E-02 & 1.034 \\
\hline 0.047 & 2113 & 0.146 E-02 & 1.059 & 0.146 E-02 & 1.059 \\
\hline 0.019 & 8321 & 0.705 E-03 & 0.823 & 0.705 E-03 & 0.823 \\
\hline
\end{tabular}

Table 1: The norm $\left\|u_{\mathfrak{M}, \delta t}-u_{\overline{\mathfrak{M}^{*}}, \delta t}\right\|_{L^{2}\left(Q_{T}\right)}$ with and without penalization term for $n=m=2$.

$\left\|u_{\mathfrak{M}, \delta t}-u_{\overline{\mathfrak{M}^{*}}, \delta t}\right\|_{L^{2}(\Omega \times(0, T))}$. One can as well check that the convergence rate is almost one.

Since the penalty term turns out to be useless numerically then we set the parameter $\gamma$ to zero in the sequel. Let us now return back to the accuracy assessment of the scheme using the exact solution (8.1). In Table 2 we list the obtained results with an isotropic tensor $\Lambda_{x x}=\Lambda_{y y}=1$. We can observe that the convergence rate of the solution is almost of second order for both kinds of meshes. We thus reach the well known order of DDFV schemes for linear problems [19, 24, 41]. Despite of being of order between 1 and 2 for linear problem, the convergence rate of the discrete gradient may be deteriorated with respect to the nonlinearity, the anisotropy and/or the discretization error. For instance we refer to [6] where the authors have found an accuracy of order 0.4 for an anisotropic Laplace equation. Here, for our nonlinear problem, we observe that the convergence rate of the gradient is close to 1 in the case of the mesh family $M 1$ whereas it is close to 2 for the Kershaw meshes. We also verify that the computed solution preserves a maximum principle property. Table 3 gives the errors in the anisotropic case where the tensor entries are $\Lambda_{x x}=1$ and $\Lambda_{y y}=0.01$. It demonstrates that the numerical solution is always nonnegative with convergence rates which are slightly similar to the isotropic case.

\begin{tabular}{|l||l|c|l|c||c||l|}
\hline \multicolumn{7}{|c|}{ M1 } \\
\hline$h_{\mathfrak{D}}$ & ERL2 & Rate & ERGL2 & Rate & $\mathrm{u}_{\min }$ & $\mathrm{u}_{\max }$ \\
\hline 0.342 & 0.127 E-01 & - & 0.324 E-01 & - & 0 & 0.703 \\
0.174 & 0.629 E-02 & 1.048 & 0.218 E-01 & 0.590 & 0 & 0.747 \\
0.092 & 0.216 E-02 & 1.669 & 0.134 E-02 & 0.755 & 0 & 0.748 \\
0.047 & 0.665 E-03 & 1.766 & 0.799 E-02 & 0.781 & 0 & 0.750 \\
0.019 & 0.126 E-03 & 1.880 & 0.345 E-02 & 0.947 & 0 & 0.750 \\
\hline \hline \multicolumn{7}{|c|}{ M2 } \\
\hline$h_{\mathfrak{D}}$ & ERL2 & Rate & ERGL2 & Rate & $\mathrm{u}_{\min }$ & $\mathrm{u}_{\max }$ \\
\hline 0.271 & 0.135 E-02 & - & 0.996 E-01 & - & 0 & 0.703 \\
0.135 & 0.369 E-03 & 1.870 & 0.265 E-01 & 1.910 & 0 & 0.744 \\
0.090 & 0.168 E-03 & 1.934 & 0.119 E-01 & 1.975 & 0 & 0.747 \\
0.067 & 0.959 E-04 & 1.954 & 0.671 E-02 & 1.990 & 0 & 0.749 \\
0.054 & 0.619 E-04 & 1.964 & 0.430 E-02 & 1.995 & 0 & 0.750 \\
\hline
\end{tabular}

Table 2: Numerical convergence with isotropic tensor and $n=m=2$.

\section{Test 2}

We now test the accuracy and the stability of our scheme thanks to the analytical solution

$$
u_{\mathrm{ex}}(x, t)=6 x_{1}^{2} \times t, \quad \forall x=\left(x_{1}, x_{2}\right) \in \Omega, \quad t \in(0, T),
$$




\begin{tabular}{|l||l|c|l|c||c||l|}
\hline \multicolumn{7}{|c|}{ M1 } \\
\hline$h_{\mathfrak{D}}$ & ERL2 & Rate & ERGL2 & Rate & $\mathrm{u}_{\min }$ & $\mathrm{u}_{\max }$ \\
\hline 0.342 & 0.130 E-01 & - & 0.327 E-01 & - & 0 & 0.736 \\
0.174 & 0.649 E-02 & 1.030 & 0.221 E-01 & 0.583 & 0 & 0.748 \\
0.092 & 0.244 E-02 & 1.529 & 0.142 E-01 & 0.695 & 0 & 0.749 \\
0.047 & 0.898 E-03 & 1.499 & 0.909 E-02 & 0.666 & 0 & 0.751 \\
0.019 & 0.180 E-03 & 1.812 & 0.416 E-02 & 0.883 & 0 & 0.750 \\
\hline \hline \multicolumn{7}{|c|}{ M2 } \\
\hline$h_{\mathfrak{D}}$ & ERL2 & Rate & ERGL2 & Rate & $\mathrm{u}_{\min }$ & $\mathrm{u}_{\max }$ \\
\hline 0.271 & 0.146 E-02 & - & 0.997 E-01 & - & 0 & 0.739 \\
0.135 & 0.402 E-03 & 1.856 & 0.267 E-01 & 1.903 & 0 & 0.746 \\
0.090 & 0.184 E-03 & 1.928 & 0.120 E-01 & 1.923 & 0 & 0.748 \\
0.067 & 0.105 E-03 & 1.948 & 0.683 E-02 & 1.967 & 0 & 0.749 \\
0.054 & 0.680 E-04 & 1.957 & 0.441 E-02 & 1.963 & 0 & 0.750 \\
\hline
\end{tabular}

Table 3: Numerical convergence with anisotropic tensor and $n=m=2$.

where the mobility function is chosen to be $f(u)=u(1-u)$. Note that this function is not a perfect square with $f(0)=f(1)=0$. This solution fulfills the continuous problem (1.1) with a corresponding source term, which is also nonnegative. It vanishes at the line $\left\{x_{1}=0\right\}$. The final time is taken as $T=0.15$. Tables 4 and 5 present the numerical convergence of the scheme including the isotropic tensor, and anisotropic one (with $\Lambda_{x x}=1$ and $\Lambda_{y y}=0.001$ ) respectively. On the mesh family $M 1$, the first table shows that the numerical scheme is accurate of almost second order whereas the second one exhibits an accuracy of order 1.5 which might be explained by the impact of anisotropy. Although the distortion of the mesh family $M_{2}$, we get a super-convergence for the solution and the gradient of its semi Kirchhoff transform. In both cases we have not recorded any undershoots nor overshoots.

\begin{tabular}{|l||l|c|l|c||c||l|}
\hline \multicolumn{7}{|c|}{ M1 } \\
\hline$h_{\mathfrak{D}}$ & ERL2 & Rate & ERGL2 & Rate & $\mathrm{u}_{\min }$ & $\mathrm{u}_{\max }$ \\
\hline 0.342 & 0.104 E-01 & - & 0.367 E-01 & - & 0 & 0.840 \\
0.174 & 0.425 E-02 & 1.335 & 0.242 E-01 & 0.622 & 0 & 0.895 \\
0.092 & 0.132 E-02 & 1.821 & 0.138 E-01 & 0.878 & 0 & 0.897 \\
0.047 & 0.365 E-03 & 1.933 & 0.696 E-02 & 1.026 & 0 & 0.900 \\
0.019 & 0.114 E-03 & 1.312 & 0.385 E-02 & 0.667 & 0 & 0.900 \\
\hline \hline \multicolumn{7}{|c|}{ M2 } \\
\hline$h_{\mathfrak{D}}$ & ERL2 & Rate & ERGL2 & Rate & $\mathrm{u}_{\min }$ & $\mathrm{u}_{\max }$ \\
\hline 0.271 & 0.124 E-02 & - & 0.102 E-00 & - & 0 & 0.882 \\
0.135 & 0.365 E-03 & 1.767 & 0.357 E-01 & 1.519 & 0 & 0.892 \\
0.090 & 0.173 E-03 & 1.849 & 0.212 E-01 & 1.286 & 0 & 0.897 \\
0.067 & 0.100 E-03 & 1.890 & 0.151 E-01 & 1.171 & 0 & 0.898 \\
0.054 & 0.654 E-04 & 1.914 & 0.118 E-01 & 1.111 & 0 & 0.900 \\
\hline
\end{tabular}

Table 4: Numerical convergence with isotropic tensor and $n=m=1$. 


\begin{tabular}{|l||l|c|l|c||c||l|}
\hline \multicolumn{7}{|c|}{ M1 } \\
\hline$h_{\mathfrak{D}}$ & ERL2 & Rate & ERGL2 & Rate & $\mathrm{u}_{\min }$ & $\mathrm{u}_{\max }$ \\
\hline 0.342 & 0.116 E-01 & - & 0.380 E-01 & - & 0 & 0.840 \\
0.174 & 0.506 E-02 & 1.245 & 0.263 E-01 & 0.547 & 0 & 0.895 \\
0.092 & 0.199 E-02 & 1.459 & 0.173 E-01 & 0.659 & 0 & 0.897 \\
0.047 & 0.754 E-03 & 1.453 & 0.108 E-01 & 0.703 & 0 & 0.900 \\
0.019 & 0.207 E-03 & 1.459 & 0.580 E-02 & 0.701 & 0 & 0.900 \\
\hline \hline \multicolumn{7}{|c|}{ M2 } \\
\hline$h_{\mathfrak{D}}$ & ERL2 & Rate & ERGL2 & Rate & $\mathrm{u}_{\min }$ & $\mathrm{u}_{\max }$ \\
\hline 0.271 & 0.210 E-02 & - & 0.118 E-00 & - & 0 & 0.881 \\
0.135 & 0.672 E-03 & 1.646 & 0.533 E-01 & 1.148 & 0 & 0.893 \\
0.090 & 0.326 E-03 & 1.783 & 0.359 E-01 & 0.974 & 0 & 0.897 \\
0.067 & 0.193 E-03 & 1.833 & 0.272 E-01 & 0.961 & 0 & 0.898 \\
0.054 & 0.127 E-03 & 1.852 & 0.220 E-01 & 0.966 & 0 & 0.900 \\
\hline
\end{tabular}

Table 5: Numerical convergence with anisotropic tensor and $n=m=1$.

\section{Test 3}

This test concerns the porous medium equation. First, we compare our scheme with the following two dimensional exact solution [17] to the main problem (1.1)

$$
u_{\mathrm{ex}}(x, t)=\frac{\lambda_{1}\left(x_{1}-0.5\right)^{2}+\lambda_{2}\left(x_{2}-0.5\right)^{2}}{1-t}, \forall x=\left(x_{1}, x_{2}\right) \in \Omega, t \in(0, T),
$$

with $\lambda_{1}=\frac{1}{16 \Lambda_{x x}}$ and $\lambda_{2}=\frac{1}{16 \Lambda_{y y}}$. The mobility function is $f(u)=2 u$. Note that this choice does not match with the assumption $\left(A_{2}\right)$. We then record the numerical convergence results in Table 6 and Table 7 with a final time set to $T=0.2$. On the first mesh sequence, one can check that the method is accurate of second order even in the presence of anisotropy $\left(\Lambda_{x x}=0.1\right.$ and $\left.\Lambda_{y y}=10\right)$. Analogous results have been observed in [17] for the same problem using a VAG (Vertex Approximate Gradient) scheme. In contrast, the super-convergence is lost in the isotropic case for the second family of meshes. This is due to the severe distortion of the mesh in the $x_{2}$-direction which yields overlapped dual grids. A similar accuracy reduction has been observed in [44], and it not caused by the absence of penalty. As expected, the second order is recovered in the anisotropic case since the contribution of the term in $x_{1}$ is less important. In any case, one can see that the method preserves the positivity.

Finally, we provide an example which exhibits a low space regularity due to the degenerate nature of the considered problem. This test has been also treated in [17] using the VAG discretization. It is about the one dimensional weak solution

$$
u_{\mathrm{ex}}(x, t)=\max \left(2 \Lambda_{x x} t-x_{1}, 0\right) \quad \forall x=\left(x_{1}, x_{2}\right), \in \Omega, t \in(0, T),
$$

to the porous medium equation (1.1) (we recall $f(u)=2 u$ ) complemented with the Dirichlet boundary condition corresponding to this exact solution. In this test-case, we consider a sequence of refined triangulations of $\Omega$ as primal meshes. We take $\Lambda_{x x}=1$ and $\Lambda_{y y}=10$. The final time is $T=0.25$. The obtained results are given in Table 8. As expected, it is shown that the discrete solution is nonnegative. It additionally converges with an order strictly less than 2 because of the anisotropy and its low regularity. This phenomenon has been also indicated in [17]. We emphasize that this loss in the convergence rate is not due to the absence of the penalization in the scheme.

\section{Test 4}

In this last test, we exhibit the efficiency of the proposed scheme using a non polynomial exact solution in space. We also illustrate the impact of the computation of the mesh size $h_{\mathcal{D}}$ on the results. To this end, 


\begin{tabular}{|l||l|c|l|c||l|}
\hline \multicolumn{7}{|c|}{ M1 } \\
\hline$h_{\mathfrak{D}}$ & ERL2 & Rate & ERGL2 & Rate & $\mathrm{u}_{\min }$ \\
\hline 0.342 & 0.426 E-03 & - & 0.945 E-02 & - & 0.206 E-03 \\
0.174 & 0.260 E-03 & 0.733 & 0.773 E-02 & 0.299 & 0.243 E-04 \\
0.092 & 0.789 E-04 & 1.860 & 0.462 E-02 & 0.803 & 0.304 E-05 \\
0.047 & 0.213 E-04 & 1.965 & 0.252 E-02 & 0.909 & 0.612 E-06 \\
0.019 & 0.450 E-05 & 1.755 & 0.115 E-02 & 0.882 & 0.234 E-06 \\
\hline \hline \multicolumn{7}{|c|}{ M2 } \\
\hline$h_{\mathfrak{D}}$ & ERL2 & Rate & ERGL2 & Rate & $\mathrm{u}_{\text {min }}$ \\
\hline 0.271 & 0.410 E-03 & - & 0.679 E-01 & - & 0.537 E-05 \\
0.135 & 0.250 E-03 & 0.713 & 0.481 E-01 & 0.495 & 0.108 E-04 \\
0.090 & 0.186 E-03 & 0.732 & 0.367 E-01 & 0.667 & 0.554 E-05 \\
0.067 & 0.149 E-03 & 0.764 & 0.294 E-01 & 0.773 & 0.739 E-06 \\
0.054 & 0.125 E-03 & 0.802 & 0.244 E-01 & 0.831 & 0.507 E-08 \\
\hline
\end{tabular}

Table 6: Numerical convergence of the scheme with $\Lambda_{x x}=\Lambda_{y y}=1$.

\begin{tabular}{|l||l|c|c|c||l|}
\hline \multicolumn{7}{|c|}{ M1 } \\
\hline$h_{\mathfrak{D}}$ & ERL2 & Rate & ERGL2 & Rate & $\mathrm{u}_{\text {min }}$ \\
\hline 0.342 & 0.340 E-01 & - & 0.103 E-00 & - & 0.734 E-03 \\
0.174 & 0.123 E-01 & 1.516 & 0.621 E-01 & 0.752 & 0.131 E-03 \\
0.092 & 0.336 E-02 & 2.022 & 0.335 E-01 & 0.963 & 0.178 E-04 \\
0.047 & 0.847 E-03 & 2.068 & 0.168 E-01 & 1.040 & 0.449 E-05 \\
0.019 & 0.222 E-03 & 1.509 & 0.884 E-02 & 0.728 & 0.234 E-06 \\
\hline \hline \multicolumn{7}{|c|}{ M2 } \\
\hline$h_{\mathfrak{D}}$ & ERL2 & Rate & ERGL2 & Rate & $\mathrm{u}_{\text {min }}$ \\
\hline 0.271 & 0.286 E-02 & - & 0.251 E-00 & - & 0.130 E-05 \\
0.135 & 0.713 E-03 & 2.004 & 0.119 E-00 & 0.987 & 0.108 E-05 \\
0.090 & 0.317 E-03 & 2.000 & 0.794 E-01 & 0.991 & 0.563 E-06 \\
0.067 & 0.179 E-03 & 1.991 & 0.598 E-01 & 0.988 & 0.739 E-07 \\
0.054 & 0.115 E-03 & 1.990 & 0.479 E-01 & 0.990 & 0.172 E-08 \\
\hline
\end{tabular}

Table 7: Numerical convergence of the scheme with $\Lambda_{x x}=0.1$ and $\Lambda_{y y}=10$.

\begin{tabular}{|l||l|c|l|c||c|}
\hline \multicolumn{7}{|c|}{ Triangular meshes } \\
\hline$h_{\mathfrak{D}}$ & ERL2 & Rate & ERGL2 & Rate & $\mathrm{u}_{\min }$ \\
\hline 0.250 & 0.176 E-01 & - & 0.165 E-00 & - & 0 \\
0.125 & 0.106 E-01 & 0.728 & 0.971 E-01 & 0.761 & 0 \\
0.063 & 0.583 E-02 & 0.865 & 0.612 E-01 & 0.667 & 0 \\
0.031 & 0.324 E-02 & 0.850 & 0.386 E-01 & 0.663 & 0 \\
0.017 & 0.177 E-02 & 0.875 & 0.242 E-01 & 0.674 & 0 \\
\hline
\end{tabular}

Table 8: Numerical convergence of the scheme with $\Lambda_{x x}=1$ and $\Lambda_{y y}=10$.

we consider an isotropic permeability tensor with $\Lambda_{x x}=\Lambda_{y y}=1$. The taken analytical solution is

$$
u_{\mathrm{ex}}(x, t)=5 t\left(\cos \left(\pi x_{1}\right)+1\right) \quad \forall x=\left(x_{1}, x_{2}\right) \in \Omega, t \in(0, T) .
$$


The mobility function is such $f(u)=u^{2}$. The final time is fixed to $T=0.1$. Inserting these data in the problem (1.1) yields a nonnegative source term. We show in Tables 9-10 the obtained results. We have considered two options to calculate $h_{\mathfrak{D}}$ : the maximum value and the averaged value of the diamond diameters. The accuracy is optimal for both cases. We observe that the errors as well as the convergence rates are sensible to the choice of mesh size computation.

\begin{tabular}{|l||l|c|l|c||c|}
\hline \multicolumn{7}{|c|}{ M1 } \\
\hline$h_{\mathfrak{D}}$ & ERL2 & Rate & ERGL2 & Rate & $\mathrm{u}_{\min }$ \\
\hline 0.342 & 0.868 E-02 & - & 0.363 E-01 & - & 0 \\
0.174 & 0.221 E-02 & 1.936 & 0.768 E-02 & 2.207 & 0 \\
0.092 & 0.682 E-03 & 1.840 & 0.229 E-02 & 1.886 & 0 \\
0.047 & 0.179 E-03 & 2.003 & 0.745 E-03 & 1.687 & 0 \\
0.019 & 0.339 E-04 & 1.862 & 0.164 E-03 & 1.703 & 0 \\
\hline \hline \multicolumn{7}{|c|}{ M2 } \\
\hline$h_{\mathfrak{D}}$ & ERL2 & Rate & ERGL2 & Rate & $\mathrm{u}_{\text {min }}$ \\
\hline 0.271 & 0.603 E-03 & - & 0.174 E-01 & - & 0 \\
0.135 & 0.187 E-03 & 1.686 & 0.424 E-02 & 2.037 & 0 \\
0.090 & 0.881 E-04 & 1.861 & 0.187 E-02 & 2.022 & 0 \\
0.067 & 0.509 E-04 & 1.908 & 0.104 E-02 & 2.015 & 0 \\
0.054 & 0.333 E-04 & 1.895 & 0.672 E-03 & 1.992 & 0 \\
\hline
\end{tabular}

Table 9: Results with maximum computation of the mesh size.

\begin{tabular}{|l||l|c|c|c||c|}
\hline \multicolumn{7}{|c|}{ M1 } \\
\hline$h_{\mathfrak{D}}$ & ERL2 & Rate & ERGL2 & Rate & $\mathrm{u}_{\min }$ \\
\hline 0.225 & 0.768 E-02 & - & 0.179 E-01 & - & 0 \\
0.126 & 0.232 E-02 & 1.739 & 0.480 E-02 & 1.916 & 0 \\
0.063 & 0.680 E-03 & 1.774 & 0.148 E-02 & 1.695 & 0 \\
0.031 & 0.179 E-03 & 1.923 & 0.584 E-03 & 1.348 & 0 \\
0.015 & 0.339 E-04 & 2.363 & 0.150 E-03 & 1.923 & 0 \\
\hline \hline \multicolumn{7}{|c|}{ M2 } \\
\hline$h_{\mathfrak{D}}$ & ERL2 & Rate & ERGL2 & Rate & $\mathrm{u}_{\min }$ \\
\hline 0.085 & 0.643 E-03 & - & 0.178 E-02 & - & 0 \\
0.042 & 0.188 E-03 & 1.792 & 0.463 E-03 & 1.966 & 0 \\
0.028 & 0.885 E-04 & 1.877 & 0.209 E-03 & 1.968 & 0 \\
0.021 & 0.512 E-04 & 1.911 & 0.119 E-03 & 1.975 & 0 \\
0.017 & 0.333 E-04 & 1.933 & 0.767 E-04 & 1.982 & 0 \\
\hline
\end{tabular}

Table 10: Results with averaged computation of the mesh size.

\section{Conclusion}

We have proposed and investigated a DDFV scheme preserving a maximum principle for nonlinear parabolic equations. The basic idea of this scheme consists in correcting the oscillatory fluxes owing to an upwind approach which is widely known in the context of hyperbolic problems. Thanks to these nonstandard approximations of the fluxes, we derived some energy estimates. The existence of solutions is proved by an inheriting result of Brouwer's fixed point theorem. With the help of some compactness properties, we have established the convergence of a sequence of approximate solutions towards the weak one of the continuous 
problem. Numerical tests showed that our scheme is accurate with optimal convergence rates and that the computed solutions are always nonnegative. Consequently, our theoretical predictions are confirmed as required. As far as we know this work is the first one which proves the discrete maximum principle together with the convergence of the numerical scheme in the context of DDFV methods.

\section{A Appendix: Technical lemmas}

Let $\mathcal{D}$ be a fixed diamond cell. We define the following $2 \times 2$ matrices

$$
\begin{aligned}
\mathbb{A}^{\mathcal{D}} & =\frac{1}{4 m_{\mathcal{D}}}\left[\begin{array}{cc}
m_{\sigma}^{2} & m_{\sigma} m_{\sigma^{*}} \\
m_{\sigma} m_{\sigma^{*}} & m_{\sigma^{*}}^{2}
\end{array}\right]=:\left[\begin{array}{cc}
\mathbb{A}_{\sigma}^{\mathcal{D}} & \mathbb{A}_{\sigma, \sigma^{*}}^{\mathcal{D}} \\
\mathbb{A}_{\sigma, \sigma^{*}}^{\mathcal{D}} & \mathbb{A}_{\sigma^{*}}^{\mathcal{D}}
\end{array}\right], \\
\mathbb{A}^{\mathcal{D}, \Lambda} & =\frac{1}{4 m_{\mathcal{D}}}\left[\begin{array}{cc}
m_{\sigma}^{2} \Lambda \mathbf{n}_{\sigma K} \cdot \mathbf{n}_{\sigma K} & m_{\sigma} m_{\sigma^{*}} \Lambda \mathbf{n}_{\sigma K} \cdot \mathbf{n}_{\sigma^{*} K^{*}} \\
m_{\sigma} m_{\sigma^{*}} \Lambda \mathbf{n}_{\sigma K} \cdot \mathbf{n}_{\sigma^{*} K^{*}} & m_{\sigma^{*}}^{2} \Lambda \mathbf{n}_{\sigma^{*} K^{*}} \cdot \mathbf{n}_{\sigma^{*} K^{*}}
\end{array}\right] \\
& =:\left[\begin{array}{cc}
\mathbb{A}_{\sigma}^{\mathcal{D}, \Lambda} & \mathbb{A}_{\sigma, \sigma^{*}}^{\mathcal{D}, \Lambda} \\
\mathbb{A}_{\sigma, \sigma^{*}}^{\mathcal{D}, \Lambda} & \mathbb{A}_{\sigma^{*}}^{\mathcal{D}, \Lambda}
\end{array}\right],
\end{aligned}
$$

and

$$
\mathbb{B}^{\mathcal{D}, \Lambda}=\left[\begin{array}{cc}
\left|\mathbb{A}_{\sigma}^{\mathcal{D}, \Lambda}\right|+\left|\mathbb{A}_{\sigma, \sigma^{*}}^{\mathcal{D}, \Lambda}\right| & 0 \\
0 & \left|\mathbb{A}_{\sigma, \sigma^{*}}^{\mathcal{D}, \Lambda}\right|+\left|\mathbb{A}_{\sigma^{*}}^{\mathcal{D}, \Lambda}\right|
\end{array}\right], \quad \forall \mathcal{D} \in \mathfrak{D} .
$$

The following lemma claims a crucial property of the matrix $\mathbb{A}^{\mathcal{D}, \Lambda}$. In particular, it states that $\mathbb{A}^{\mathcal{D}, \Lambda}$ is positive definite.

Lemma A.1. [19] There exist some positive constants $\lambda_{0}$ and $\lambda_{1}$ depending only on the mesh regularity and on $\underline{\Lambda}, \bar{\Lambda}$ satisfying

$$
\begin{aligned}
& \mathbb{A}^{\mathcal{D}, \Lambda} x \cdot x \leq \mathbb{B}^{\mathcal{D}, \Lambda} x \cdot x \leq \lambda_{1} \mathbb{A}^{\mathcal{D}, \Lambda} x \cdot x, \quad \forall x \in \mathbb{R}^{2}, \\
& \lambda_{0} \mathbb{A}^{\mathcal{D}} x \cdot x \leq \mathbb{A}^{\mathcal{D}, \Lambda} x \cdot x, \quad \forall x \in \mathbb{R}^{2} .
\end{aligned}
$$

Lemma A.2. Consider the following piecewise constant functions

$$
\begin{array}{ll}
\bar{\xi}_{\mathcal{D}}^{n+1}:=\max _{M \in \mathcal{V}_{\mathcal{D}}}\left\{\xi\left(u_{M}^{n+1}\right)\right\}, & \underline{\xi}_{\mathcal{D}}^{n+1}:=\min _{M \in \mathcal{V}_{\mathcal{D}}}\left\{\xi\left(u_{M}^{n+1}\right)\right\}, \\
\bar{\xi}_{\mathcal{T}, \delta t \mid \mathcal{D} \times\left(t^{n}, t^{n+1}\right]}:=\bar{\xi}_{\mathcal{D}}^{n+1}, & \underline{\xi}_{\mathcal{T}, \delta t} \mid \mathcal{D} \times\left(t^{n}, t^{n+1}\right]:=\underline{\xi}_{\mathcal{D}}^{n+1},
\end{array}
$$

where we denote $\mathcal{V}_{\mathcal{D}}=\left\{K, L, K^{*}, L^{*}\right\}$. Then

$$
\lim _{h_{\mathfrak{D}}, \delta t \rightarrow 0}\left\|\bar{\xi}_{\mathcal{T}, \delta t}-\underline{\xi}_{\mathcal{T}, \delta t}\right\|_{L^{2}\left(Q_{T}\right)}=0
$$

Proof. The proof extends similar ideas provided in [20].

Acknowledgment. The authors would like to thank the anonymous reviewer for his valuable comment and some precise suggestions that improved the presentation and the quality of this work.

\section{References}

[1] M. Afif and B. Amaziane. Convergence of finite volume schemes for a degenerate convection-diffusion equation arising in flow in porous media. Computer methods in applied mechanics and engineering, 191(46):5265-5286, 2002. 
[2] H. W. Alt and S. Luckhaus. Quasilinear elliptic-parabolic differential equations. Mathematische Zeitschrift, 183(3):311-341, 1983.

[3] B. Andreianov, M. Bendahmane, and F. Hubert. On 3D DDFV discretization of gradient and divergence operators: discrete functional analysis tools and applications to degenerate parabolic problems. Computational Methods in Applied Mathematics, 13(4):369-410, 2013.

[4] B. Andreianov, M. Bendahmane, F. Hubert, and S. Krell. On 3D DDFV discretization of gradient and divergence operators. I. Meshing, operators and discrete duality. IMA Journal of Numerical Analysis, 32(4):1574-1603, 2012.

[5] B. Andreianov, M. Bendahmane, and M. Saad. Finite volume methods for degenerate chemotaxis model. Journal of computational and applied mathematics, 235(14):4015-4031, 2011.

[6] B. Andreianov, F. Boyer, and F. Hubert. Discrete duality finite volume schemes for Leray- Lionstype elliptic problems on general 2D meshes. Numerical Methods for Partial Differential Equations, 23(1):145-195, 2007.

[7] B. A. Andreianov, M. Gutnic, and P. Wittbold. Convergence of finite volume approximations for a nonlinear elliptic-parabolic problem: A" continuous" approach. SIAM Journal on Numerical Analysis, 42(1):228-251, 2004.

[8] O. Angelini, K. Brenner, and D. Hilhorst. A finite volume method on general meshes for a degenerate parabolic convection-reaction-diffusion equation. Numerische Mathematik, 123(2):219-257, 2013.

[9] J. W. Barrett and P. Knabner. Finite element approximation of the transport of reactive solutes in porous media. Part II: Error estimates for equilibrium adsorption processes. SIAM journal on numerical analysis, 34(2):455-479, 1997.

[10] M. Bendahmane, Z. Khalil, and M. Saad. Convergence of a finite volume scheme for gas-water flow in a multi-dimensional porous medium. Mathematical Models and Methods in Applied Sciences, 24(01):145$185,2014$.

[11] M. Bessemoulin-Chatard, C. Chainais-Hillairet, and F. Filbet. On discrete functional inequalities for some finite volume schemes. IMA Journal of Numerical Analysis, 35(3):1125-1149, 2014.

[12] M. Bessemoulin-Chatard and F. Filbet. A finite volume scheme for nonlinear degenerate parabolic equations. SIAM Journal on Scientific Computing, 34(5):B559-B583, 2012.

[13] H. Brezis. Functional analysis, Sobolev spaces and partial differential equations. Springer Science \& Business Media, 2010.

[14] J.-S. Camier and F. Hermeline. A monotone nonlinear finite volume method for approximating diffusion operators on general meshes. International Journal for Numerical Methods in Engineering, 107(6):496$519,2016$.

[15] C. Cancès, M. Cathala, and C. Le Potier. Monotone corrections for generic cell-centered finite volume approximations of anisotropic diffusion equations. Numerische Mathematik, 125(3):387-417, 2013.

[16] C. Cancès and C. Guichard. Convergence of a nonlinear entropy diminishing control volume finite element scheme for solving anisotropic degenerate parabolic equations. Mathematics of Computation, 85(298):549-580, 2016.

[17] C. Cancès and C. Guichard. Numerical analysis of a robust free energy diminishing finite volume scheme for parabolic equations with gradient structure. Foundations of Computational Mathematics, 17(6):1525-1584, 2017.

[18] C. Cancès, M. Ibrahim, and M. Saad. Positive nonlinear CVFE scheme for degenerate anisotropic Keller-Segel system. SMAI Journal of Computational Mathematics, 3:1-28, 2017. 
[19] Cancès, Clément and Guichard, Cindy. Numerical analysis of a robust free energy diminishing finite volume scheme for parabolic equations with gradient structure. Foundations of Computational Mathematics, 17(6):1525-1584, 2017.

[20] C. Chainais-Hillairet, S. Krell, and A. Mouton. Convergence analysis of a DDFV scheme for a system describing miscible fluid flows in porous media. Numerical Methods for Partial Differential Equations, 31(3):723-760, 2015.

[21] G. Chavent and J. Jaffré. Mathematical models and finite elements for reservoir simulation: single phase, multiphase and multicomponent flows through porous media, volume 17. Elsevier, 1986.

[22] Y. Coudière and F. Hubert. A 3D discrete duality finite volume method for nonlinear elliptic equations. SIAM Journal on Scientific Computing, 33(4):1739-1764, 2011.

[23] Y. Coudière and G. Manzini. The discrete duality finite volume method for convection-diffusion problems. SIAM Journal on Numerical Analysis, 47(6):4163-4192, 2010.

[24] S. Delcourte, K. Domelevo, and P. Omnès. Discrete duality finite volume method for second order elliptic problems. Hermes Science publishing, pages 447-458, 2005.

[25] K. Domelevo and P. Omnès. A finite volume method for the Laplace equation on almost arbitrary two-dimensional grids. ESAIM: Mathematical Modelling and Numerical Analysis, 39(6):1203-1249, 2005.

[26] J. Droniou. Finite volume schemes for diffusion equations: introduction to and review of modern methods. Mathematical Models and Methods in Applied Sciences, 24(08):1575-1619, 2014.

[27] J. Droniou, R. Eymard, T. Gallouët, C. Guichard, and R. Herbin. The gradient discretisation method. ESAIM: Mathematical Modelling and Numerical Analysis, 50(3):749-781, 2016.

[28] C. Ebmeyer. Error estimates for a class of degenerate parabolic equations. SIAM journal on numerical analysis, 35(3):1095-1112, 1998.

[29] L. C. Evans. Partial differential equations. 2nd edn. Amer. Math. Soc., 2010.

[30] R. Eymard, P. Féron, T. Gallouët, R. Herbin, and C. Guichard. Gradient schemes for the Stefan problem. International Journal On Finite Volumes, 10, 2013.

[31] R. Eymard, T. Gallouët, and R. Herbin. Finite volume methods. Handbook of numerical analysis, 7:713-1018, 2000.

[32] R. Eymard, T. Gallouët, R. Herbin, and A. Michel. Convergence of a finite volume scheme for nonlinear degenerate parabolic equations. Numerische Mathematik, 92(1):41-82, 2002.

[33] R. Eymard, T. Gallouët, D. Hilhorst, and Y. N. Slimane. Finite volumes and nonlinear diffusion equations. ESAIM: Mathematical Modelling and Numerical Analysis, 32(6):747-761, 1998.

[34] R. Eymard, D. Hilhorst, and M. Vohralík. A combined finite volume-nonconforming/mixed-hybrid finite element scheme for degenerate parabolic problems. Numerische Mathematik, 105(1):73-131, 2006.

[35] F. Filbet. A finite volume scheme for the Patlak-Keller-Segel chemotaxis model. Numerische Mathematik, 104(4):457-488, 2006.

[36] G. Gagneux and M. Madaune-Tort. Unicité des solutions faibles d' équations de diffusion-convection. Comptes rendus de l'Académie des sciences. Série 1, Mathématique, 318(10):919-924, 1994.

[37] Z. Gao and J. Wu. A second-order positivity-preserving finite volume scheme for diffusion equations on general meshes. SIAM Journal on Scientific Computing, 37(1):A420-A438, 2015. 
[38] M. Ghilani, E. H. Quenjel, and M. Saad. Positive control volume finite element scheme for a degenerate compressible two-phase flow in anisotropic porous media. Computational Geosciences, 23(1):55-79, 2019 .

[39] R. Herbin and F. Hubert. Benchmark on discretization schemes for anisotropic diffusion problems on general grids. In R. Eymard and J.-M. Herard, editors, Finite Volumes for Complex Applications V, pages 659-692. Wiley, 2008.

[40] F. Hermeline. Une méthode de volumes finis pour les équations elliptiques du second ordre. Comptes Rendus de l'Académie des Sciences-Series I-Mathematics, 326(12):1433-1436, 1998.

[41] F. Hermeline. A finite volume method for the approximation of diffusion operators on distorted meshes. Journal of computational Physics, 160(2):481-499, 2000.

[42] W. Jäger and J. Kačur. Solution of porous medium type systems by linear approximation schemes. Numerische Mathematik, 60(1):407-427, 1991.

[43] K. Karlsen, N. Risebro, and J. Towers. Upwind difference approximations for degenerate parabolic convection-diffusion equations with a discontinuous coefficient. IMA journal of numerical analysis, $22(4): 623-664,2002$.

[44] S. Krell. Schémas Volumes Finis en mécanique des fluides complexes. PhD thesis, Université de Provence-Aix-Marseille I, 2010.

[45] S. Krell and G. Manzini. The Discrete Duality Finite Volume method for the Stokes equations on 3-D polyhedral meshes. SIAM Journal on Numerical Analysis, 50(2):808-837, 2012.

[46] R. Lazarov, I. D. Mishev, and P. S. Vassilevski. Finite volume methods for convection-diffusion problems. SIAM Journal on Numerical Analysis, 33(1):31-55, 1996.

[47] K. Lipnikov, M. Shashkov, D. Svyatskiy, and Y. Vassilevski. Monotone finite volume schemes for diffusion equations on unstructured triangular and shape-regular polygonal meshes. Journal of Computational Physics, 227(1):492-512, 2007.

[48] R. Nochetto, A. Schmidt, and C. Verdi. A posteriori error estimation and adaptivity for degenerate parabolic problems. Mathematics of Computation of the American Mathematical Society, 69(229):1-24, 2000 .

[49] S. Osher and F. Solomon. Upwind difference schemes for hyperbolic systems of conservation laws. Mathematics of computation, 38(158):339-374, 1982.

[50] J. L. Vázquez. The porous medium equation: mathematical theory. Oxford University Press, 2007.

[51] G. Yuan and Z. Sheng. Monotone finite volume schemes for diffusion equations on polygonal meshes. Journal of computational physics, 227(12):6288-6312, 2008. 